\title{
Circumstellar disks around Herbig Be stars
}

\author{
T. Alonso-Albi ${ }^{1}$, A. Fuente ${ }^{1}$, R. Bachiller ${ }^{1}$, R. Neri ${ }^{2}$, P. Planesas ${ }^{1,3}$, L. Testi ${ }^{4,6}$, O. Berné ${ }^{5}$, and C. Joblin ${ }^{5}$ \\ 1 Observatorio Astronómico Nacional, Apdo. 112, 28803 Alcalá de Henares (Madrid), Spain \\ e-mail: t.alonso@oan.es \\ 2 Institut de Radio Astronomie Milimetrique, 300 rue de la Piscine, Domaine Universitaire de Grenoble, \\ 38406 St. Martin d'Hères, France \\ 3 Atacama Large Millimeter/Submillimeter Array, Joint ALMA Office, Santiago, Chile \\ 4 INAF - Osservatorio Astrofisico de Arcetri, Largo Enrico Fermi 5, 50125 Firenze, Italy \\ 5 Centre d'Étude Spatiale des Rayonnements, CNRS et Université Paul Sabatier Toulouse 3, Observatoire Midi-Pyrénées, \\ 9 Av. du Colonel Roche, 31028 Toulouse Cedex 04, France \\ ${ }^{6}$ European Southern Observatory, Karl Schwarzschild str. 2, 85748 Garching, Germany
}

Received 17 June 2008 / Accepted 8 December 2008

\begin{abstract}
Aims. Our goal is to investigate the properties of the circumstellar disks around intermediate mass stars to determine their occurrence, lifetime and evolution.

Methods. We completed a search for circumstellar disks around Herbig Be stars using the NRAO Very Large Array (VLA) and the IRAM Plateau de Bure (PdB) interferometers. Thus far, we have observed 6 objects with 4 successful detections. The results towards 3 of these stars (R Mon, MWC 1080, MWC 137) were presented elsewhere. We present our new VLA and PdBI data for the three objects MWC 297, Z CMa, and LKH $\alpha 215$. We constructed the SED from near-IR to centimeter wavelengths by adding our millimeter and centimeter data to the available data at other wavelengths, mainly Spitzer images. The entire SED was fitted using a disk+envelope model. In addition, we compiled all the disk millimeter observations in the literature and completed a statistical analysis of all the data.

Results. We show that the disk mass is usually only a small percentage (less than 10\%) of the mass of the entire envelope in HBe stars. For the disks, there are large source-to-source variations. Two disks in our sample, R Mon and Z CMa, have similar sizes and masses to those found in T Tauri and Herbig Ae stars. The disks around MWC 1080 and MWC 297 are, however, smaller $\left(r_{\text {out }}<100\right.$ AU). We did not detect the disks towards MWC 137 and $\mathrm{LkH} \alpha 215$ at millimeter wavelengths, which limits the mass and the size of the possible circumstellar disks.

Conclusions. A comparison between our data and previous results for $\mathrm{T}$ Tauri and Herbig Ae stars indicates that although massive disks $\left(\sim 0.1 M_{\odot}\right)$ are found in young objects $\left(\sim 10^{4} \mathrm{yr}\right)$, the masses of the disks around Herbig Be stars are usually $5-10$ times lower than those around lower mass stars. We propose that disk photoevaporation is responsible for this behavior. In Herbig Be stars, the UV radiation disperses the gas in the outer disk on a timescale of a few $10^{5} \mathrm{yr}$. Once the outer part of the disk has vanished, the entire gaseous disk is photoevaporated on a very short timescale $\left(\sim 10^{5} \mathrm{yr}\right)$ and only a small, dusty disk consisting of large grains remains.
\end{abstract}

Key words. stars: formation - stars: pre-main sequence - circumstellar matter - planetary systems: formation planetary systems: protoplanetary disks

\section{Introduction}

Herbig $\mathrm{Ae} / \mathrm{Be}(\mathrm{HAeBe})$ stars are intermediate-mass $(M \sim$ 2-8 $M_{\odot}$ ) pre-main-sequence objects. Since these objects share many characteristics with high-mass stars (clustering, PDRs), but are far closer to us and less embedded, the detection of circumstellar disks around these stars is crucial to the understanding of the massive-star-formation process. Herbig Ae (HAe) stars are the precursors of Vega-type systems: determining the frequency and timescales of the disks around HAe stars is therefore also important for planet formation studies.

Substantial theoretical and observational efforts have been carried out recently for the understanding of the disk occurrence and evolution in HAeBe stars (Meeus et al. 2001; Vink et al. 2002; Millan-Gabet et al. 2001; Acke et al. 2005). On the basis of the different mid-infrared excesses, Meeus et al. (2001) proposed that disks around Herbig $\mathrm{Ae} / \mathrm{Be}$ stars can be classified into two Groups that they interpreted in terms of different geometries: Group I sources with strong mid-infrared $(20-100 \mu \mathrm{m})$ flux excesses and Group II sources with modest infrared excesses. Group I sources have Chiang \& Goldreich-like flared disks and Group II sources host flatter, self-shadowed disks. Leinert et al. (2004) confirmed this hypothesis on the basis of $10 \mu \mathrm{m}$ interferometry data. While most HAe stars belong to Group I and are surrounded by disks similar to those in T Tauri (TT) stars, Herbig $\mathrm{Be}(\mathrm{HBe})$ stars are mostly found in Group II and seem to be surrounded by disks with a flatter geometry (Acke et al. 2005).

Although the existence of different types of disks is well accepted, whether this difference is due to the different characteristics of the central star (hotter stars produce flatter disks), or to different characteristics of the circumstellar matter (different grain opacities), or to an evolutionary link between flared, selfshadowed, and debris disks is not established. If the evolutionary scenario stands, the different geometry between disks in HAe and $\mathrm{HBe}$ stars is the consequence of a more rapid grain growth and a shorter dissipation timescale in the latter. The grain growth causes the optical depth of the disk to decrease and allows the 
UV radiation to penetrate deep into the circumstellar disk and photoevaporate the disk external layers (Dullemond \& Dominik 2004). Disks around HBe would flatten and lose a large fraction of their mass before the pre-main-sequence phase $(<0.1 \mathrm{Myr})$.

One problem in discerning between the different scenarios is that most studies are based on the optical-NIR and mid-IR observations. Optical-NIR and mid-IR observations probe the region within a few AU around the star. In addition, since the disk is optically thick at these wavelengths, they only provide information about the disk surface and cannot be used to derive the disk mass. An obvious step towards the formation of planetesimals in disks is coagulation and growth of the submicron sized grains accreted from the proto-stellar envelope (Dominik et al. 2007). Although the end results are plainly visible in our own planetary system, the details and mechanisms of dust evolution are not understood. The main problem is that the cooler, large grains do not emit significantly at infrared wavelengths. Only by observing the submm-mm range of the spectrum are we able to determine the sizes and properties of these large grains. Millimeter observations must be used to trace this more evolved grain component. Thus, imaging disks at $\mathrm{mm}$ wavelengths is required to trace the outer part of the disk and determine its mass and grain properties.

We have carried out a search for circumstellar disks around HBe stars using the NRAO Very Large Array (VLA) and the IRAM Plateau de Bure (PdB) interferometers to investigate the properties of the circumstellar disks around intermediate-mass stars and to determine eventually their occurence, lifetime, and evolution. Some results have already been published elsewhere (Fuente et al. 2003, 2006; Alonso-Albi et al. 2007; Alonso-Albi et al. 2008). In this Paper, we present new observational data (LkH $\alpha$ 215, Z CMa and MWC 297) that complete our survey and we provide a comprehensive analysis of all the data obtained thus far.

\section{Observations}

\subsection{Selected sample}

The list of observed sources is shown in Table 1. Our sample was built according to the following criteria: firstly, we included most well-studied Herbig stars with spectral type close to B0 in the northern hemisphere (R Mon, MWC 1080, MWC 137, MWC 297). In a pioneering work, Natta et al. (2000) compiled the existing $1.3 \mathrm{~mm} / 2.7 \mathrm{~mm}$ interferometric disk detections in $\mathrm{HAeBe}$ stars. By this time, only one disk had been detected in stars with spectral type earlier than B7-8. They proposed that this lack of detections could be due to a shorter dissipation timescale for the circumstellar disk in stars of spectral type earlier than B7. If the disk mass decreases with stellar spectral type, one expects to measure the minimum disk masses towards the most massive B0 stars. Secondly, we included some objects with spectral types between B7 and B8, where the transition between the TT-like and massive star disks, if it exists, is expected to occur. Additional criteria were the closeness to the Sun and previous detections with single-dish telescopes.

\subsection{Millimeter and centimeter observations}

Thus far, we have observed $6 \mathrm{HBe}$ stars with 4 successful detections. The VLA and PdBI observations towards R Mon, MWC 1080, and MWC 137 were already reported by Fuente et al. (2003, 2006). In this Paper, we present new VLA observations towards MWC 297 and Z CMa, and new PdBI observations
Table 1. Sample of observed Herbig Be stars.

\begin{tabular}{llllll}
\hline \hline Name & $\begin{array}{l}\text { RA } \\
(\mathrm{J} 2000)\end{array}$ & $\begin{array}{l}\text { Dec } \\
(\mathrm{J} 2000)\end{array}$ & $\begin{array}{l}\text { Dist. } \\
(\mathrm{pc})\end{array}$ & $\begin{array}{l}\text { Mass } \\
\left(M_{\odot}\right)\end{array}$ & $\begin{array}{l}\text { Sp. } \\
\text { Type }\end{array}$ \\
\hline MWC 137 & $06: 18: 45.50$ & $15: 16: 52.4$ & 1300 & 14 & B0 \\
R Mon & $06: 39: 09.95$ & $08: 44: 10.7$ & 800 & 8 & B0 \\
MWC 1080 & $23: 17: 25.57$ & $60: 50: 43.3$ & 1000 & 10 & B0 \\
MWC 297 & $18: 27: 39.53$ & $-03: 49: 52.0$ & 250 & 9 & B1.5 \\
LKH $\alpha$ 215 & $06: 32: 41.79$ & $10: 09: 33.6$ & 800 & 7 & B7.5 \\
Z CMa & $07: 03: 43.16$ & $-11: 33: 06.2$ & 930 & 12 & B8 \\
\hline
\end{tabular}

towards $\mathrm{LkH} \alpha 215, \mathrm{Z}$ CMa and MWC 297. A summary of these observations, wavelength, beam, and date, is shown in Table 2.

The data were calibrated, mapped, and analyzed in the GILDAS software package. Continuum images at $\mathrm{mm}$ wavelengths have been produced by averaging the channels free of line emission after careful visual inspection. Natural weightings were applied to the measured visibilities producing the beams shown in Table 2. Since the disks are expected to be unresolved by our interferometric observations, we used the peak flux to construct the SED. The measured peak fluxes are shown in Table 3. The rms of the images are shown in Tables A.1, A.3, A.5, A.7, A.9 and A.11.

\subsection{Mid-IR observations}

Four of the 6 sources in our sample have been observed at $24 \mu \mathrm{m}$ either with MIPS or IRS onboard the Spitzer Space Telescope, namely MWC 1080, MWC 297, R Mon, and LkH $\alpha$ 215. We retrieved the post-basic-calibrated data (pbcd) for imagery and basic-calibrated data for spectroscopy (bcd). The pbcd files were used without additional processing. The bcd spectral mapping files were processed with the CUBISM software (Smith et al. 2007).

\section{Results}

\subsection{Interferometric data}

The results of our observations are shown in Table 3 . We detected emission at $1.3 \mathrm{~mm}$ and/or $2.7 \mathrm{~mm}$ towards 5 out of the 6 stars studied. Emission at centimeter $(\mathrm{cm})$ wavelengths using the VLA array was detected towards Z CMa, MWC 297, MWC 137, and MWC 1080. In these massive stars, a significant fraction of the $1.3 \mathrm{~mm}$ flux can be attributed to free-free emission instead of dust, thermal emission arising in the circumstellar disk. To estimate this contribution, we fitted the free-free spectral index at $\mathrm{cm}$ wavelengths and extrapolated the emission to millimeter (mm) wavelengths. In the case of MWC 137, all the flux seems to be due to free-free emission without any significant excess at $1.3 \mathrm{~mm}$ and $2.7 \mathrm{~mm}$. In the other cases, there is some flux excess at $\mathrm{mm}$ wavelengths that can be interpreted as arising in a circumstellar disk. We show in Table 3 the spectral index between $1.3 \mathrm{~mm}$ and $2.7 \mathrm{~mm}$ interferometric observations. Two values are presented: the first represents the total observed fluxes and the second with the free-free emission substracted, i.e., the spectral index of the thermal, dust emission. The $1.3 \mathrm{~mm} / 2.7 \mathrm{~mm}$ spectral index is quite low $(<2.2)$ for all sources. As discussed in the following sections, this low spectral index constitutes observational evidence of either optically thick dust emission or the presence of large grains in the disk midplane (see e.g. Alonso-Albi et al. 2008). As we discuss in Sect. 5.7, the 
Table 2. Observations.

\begin{tabular}{lllll}
\hline \hline Name & $\lambda(\mathrm{mm})$ & Beam size & Date & Telescope \\
\hline MWC 297 & 1.3 & $1.1^{\prime \prime} \times 0.4^{\prime \prime}$ & Feb. 2006 & PdBI \\
& 2.6 & $1.4^{\prime \prime} \times 0.9^{\prime \prime}$ & Feb. 2006 & \\
LKH $\alpha$ 215 & 1.3 & $3.3^{\prime \prime} \times 1.8^{\prime \prime}$ & Apr. 2002 & PdBI \\
& 2.6 & $6.8^{\prime \prime} \times 3.7^{\prime \prime}$ & Apr. 2002 & \\
Z CMa & 1.3 & $2.3^{\prime \prime} \times 0.9^{\prime \prime}$ & Jan./Mar. 2006 & PdBI \\
& 2.6 & $4.1^{\prime \prime} \times 2.0^{\prime \prime}$ & Jan./Mar. 2006 & \\
MWC 297 & 7 & $1.9^{\prime \prime} \times 1.6^{\prime \prime}$ & Dec. 2005 & VLA \\
& 13 & $3.9^{\prime \prime} \times 3.1^{\prime \prime}$ & Dec. 2005 & \\
Z CMa & 7 & $1.4^{\prime \prime} \times 1.4^{\prime \prime}$ & Oct./Dec. 2005 & VLA \\
& 13 & $3.5^{\prime \prime} \times 2.7^{\prime \prime}$ & Oct./Dec. 2005 & \\
& 36 & $9.2^{\prime \prime} \times 6.6^{\prime \prime}$ & Oct./Dec. 2005 & \\
\hline
\end{tabular}

second possibility is the most plausible in the circumstellar disks around $\mathrm{R}$ Mon and $\mathrm{Z}$ CMa.

In Table 3, we also show the ratio of our interferometric fluxes to previous single-dish data. In most cases, the flux detected by interferometric techniques is far lower $(<10 \%)$ than that detected by single-dish observations. This implies that the envelope emission dominates the single-dish flux at FIR and mm wavelengths in most $\mathrm{HBe}$ stars. For this reason, interferometric measurements are essential to estimating the true disk emission. Since interferometric observations are unavailable at all wavelengths, a disk+envelope model is required to fit the observed SED properly.

\subsection{Observed SED}

We reconstructed the entire SED towards our sample by completing our VLA, PdBI, and Spitzer photometry with the data available in the literature. At optical and NIR wavelengths, observations are heavily affected by extinction. To correct for this reddening, we substracted the standard $B-V$ color corresponding to the spectral type of each star, as given by Johnson (1966), to the observed $B-V$ color. This color excess $E$ was used to calculate the visual extinction $A_{\mathrm{v}}=R_{v}$ E assuming a ratio of total-to-selective absorption of $R_{\mathrm{V}}=3.1$ (Cardelli et al. 1989). With these two parameters, we estimate the extinction corresponding to a particular observation using the parameterized extinction law of Cardelli et al. (1989), which can be applied to wavelengths between 0.12 and $3.5 \mu \mathrm{m}$. The complete photometric set of values with the observed and dereddened fluxes for each source is presented in Appendix A.

Most HAeBe stars are well-known variable stars, a problem that is relevant to our case because we used photometry recovered from the literature in the past decades. The variability affects the $B-V$ color and produces some additional uncertainty in the extinction estimates.

\section{Model}

As mentioned in Sect. 3.1, a two-component model is required to fit the observed SEDs. The disks were fitted using a passive, irradiated circumstellar disk model described by Alonso-Albi et al. (2008) allowing us to consider different grain populations for the midplane and surface layers. Grain populations were characterized by a silicate/graphite mixture, the maximum grain size $\left(a_{\max }\right)$, and the slope of the grain size distribution $(p)$. The value of $p$ was fixed to 3.5 and a standard grain mixture (SM: $86 \%$ silicate/14\% graphite) were assumed for the midplane and surface layers. In two cases, R Mon and Z CMa, we were unable to obtain a reliable fitting with these assumptions and we needed to vary the grain mixture in the surface layer. These cases are discussed in detail in Sect. 5.7.

In the fitting process, we consider that the emission at wavelengths shorter than $10 \mu \mathrm{m}$ and the fluxes measured by interferometric techniques (PdBI,VLA) arise only in the star/disk system. This is based on the folowing scientific arguments: firstly, the dust emitting at $\leq 10 \mu \mathrm{m}$ has a temperature of $>300 \mathrm{~K}$. Assuming that the star is the only heating source in the region, the dust should be located at radii $<100-300$ AU, which is the typical size of a circumstellar disk. Secondly, the spatial resolution of our interferometric $\mathrm{mm}$ and $\mathrm{cm}$ observations is usually below 1000 AU. It is, again, reasonable to consider that most of the emission detected with the interferometers arise in the disk, although a contribution from the envelope may also exist in some cases. The emission observed at mid-IR and FIR wavelengths (Spitzer, SCUBA) are, however, assumed to be the sum of the disk and the envelope components. This is consistent with the MIPS images at $24 \mu \mathrm{m}$, which show nebulous emission around MWC 1080, MWC 297, R Mon, and LkH $\alpha 215$.

The envelope was modeled using a ray-tracing code. The dust mass, density law, and geometry were determined by the fitting procedure. Our aim was to fit the observed emission and the visual extinction towards the star. Since the visual extinction was determined by the amount of mass along the line-of-sight, it constrains the geometry of the envelope. As discussed in Sect. 5, the morphology of the continuum submillimeter and millimeter maps were also taken into account in constraining the envelope geometry. We modeled each envelope by considering two possible geometries, a sphere and a toroid. The dust temperature was assumed to be described by a power law, $T(r)=T_{\mathrm{o}}\left(r / r_{\mathrm{o}}\right)^{-0.5}$, where the values of $T_{\mathrm{o}}$ and $r_{\mathrm{o}}$ depends on whether the envelope was shadowed or not by the inner circumstellar disk. In the nonshadowed case, typical of a sphere geometry, the effective temperature and radius of the star were used as values for $T_{\mathrm{o}}$ and $r_{\mathrm{o}}$. In the shadowed case, typical of toroidal envelopes, we extrapolated the dust temperature in the disk midplane. The dust opacities in the envelope were assumed to be those reported by Ossenkopf \& Henning (1994) for protostellar cores, using the standard MRN dust model with icy mantles. The resulting emission was convolved by the beam of the observations before being compared with the observed SED.

The goodness of fit was estimated using a dispersion parameter $(\chi)$ defined to be $\chi=\sqrt{\sum\left(\left(F_{\text {observed }}-F_{\text {modeled }}\right) / F_{\text {observed }}\right)^{2} / n}$, where $F$ is the flux and the sum is extended for all detections in the SED dominated by the disk emission from $2 \mu \mathrm{m}$ and beyond, and $n$ the number of detections. In Table 4, we show the best-fit (minimum value of $\chi$ ) solution for each source.

\section{Individual sources}

\subsection{R Mon}

The SED towards R Mon is the only in our sample for which the first peak occurs at 4-5 $\mu \mathrm{m}$ instead of at $2 \mu \mathrm{m}$ (see Fig. 1). As a result, the SED can be fitted by a Chiang \& Goldreichlike model without the presence of an inner rim. However, an inner rim cannot be discarded because we lack of photometry between $2 \mu \mathrm{m}$ and $3 \mu \mathrm{m}$, and extinction at these wavelengths is very high. The near and mid-IR part of the SED is well fitted by the emission of a circumstellar disk with $r_{\text {out }} \sim 150 \mathrm{AU}$. The 
Table 3. Summary of observational results

\begin{tabular}{|c|c|c|c|c|c|c|c|}
\hline Name & $\begin{array}{l}3.6 \mathrm{~cm} \\
(\mathrm{mJy} / \text { beam })\end{array}$ & $\begin{array}{l}1.3 \mathrm{~cm} \\
(\mathrm{mJy} / \text { beam })\end{array}$ & $\begin{array}{l}7 \mathrm{~mm} \\
(\mathrm{mJy} / \text { beam })\end{array}$ & $\begin{array}{l}2.7 \mathrm{~mm} \\
(\mathrm{mJy} / \mathrm{beam})\end{array}$ & $\begin{array}{l}1.3 \mathrm{~mm} \\
(\mathrm{mJy} / \text { beam })\end{array}$ & $\begin{array}{l}\text { Spec. index }{ }^{1} \\
(1.3-2.6 \mathrm{~mm})\end{array}$ & $\begin{array}{l}F_{\mathrm{PdBI}} / F_{\mathrm{JCMT}}^{2} \\
(1.3 \mathrm{~mm})\end{array}$ \\
\hline MWC 137 & & 0.9 & 1.3 & 4.1 & 7.1 & $0.79 /-$ & 0.075 \\
\hline R Mon & & 0.9 & 1.3 & 4.1 & 11.8 & $1.53 / 2.14$ & 0.15 \\
\hline MWC 1080 & & & & $<1.7$ & 3.1 & $>0.87 />1.02$ & 0.013 \\
\hline MWC 297 & & 23 & 29 & 149 & 175 & $1.01^{3} / 1.31^{3}$ & 0.39 \\
\hline $\mathrm{LkH} \alpha 215$ & & & & $<0.6$ & $<1.5$ & $-1-$ & $<0.026$ \\
\hline Z CMa & 2.2 & 2.1 & 2.1 & 8.5 & 26 & $1.61 / 1.88$ & 0.037 \\
\hline
\end{tabular}

${ }^{1}$ The $1.3 \mathrm{~mm}-2.7 \mathrm{~mm}$ spectral index before (left) and after (right) subtracting the free-free emission; ${ }^{2}$ see references in Appendix A; ${ }^{3}$ beam dependent, see Sect. 5.3.

Table 4. Disk modeling results ${ }^{1}$.

\begin{tabular}{|c|c|c|c|c|c|c|c|c|}
\hline Name & $\begin{array}{l}\text { Dust } \\
\text { Mass }\left(M_{\odot}\right)\end{array}$ & $\begin{array}{l}\text { Inner radius } \\
\text { (AU) }\end{array}$ & $\begin{array}{l}\operatorname{Rim} T \\
(\mathrm{~K})\end{array}$ & $\begin{array}{l}\text { Rim height } \\
\text { (AU) }\end{array}$ & $\begin{array}{l}\text { Density } \\
\text { slope }\end{array}$ & $\begin{array}{l}\text { Inclination } \\
\text { (degrees) }\end{array}$ & $\begin{array}{l}\text { Outer radius } \\
\text { (AU) }\end{array}$ & Dispersion \\
\hline MWC 137 & $1.0 \times 10^{-5} \begin{array}{r}2 \times 10^{-4} \\
5 \times 10^{-6}\end{array}$ & 8.4 & & & $-1-$ & $80 \frac{90}{70}$ & $18_{-}^{25}$ & 0.20 \\
\hline R Mon & $1.4 \times 10^{-4} \begin{array}{c}2 \times 10^{-4} \\
9 \times 10^{-5}\end{array}$ & 18.2 & & & $-0.45_{-1.0}^{0.0}$ & $60 \begin{array}{l}70 \\
40\end{array}$ & $150_{100}^{200}$ & 0.09 \\
\hline MWC 1080 & $5.0 \times 10^{-5} \begin{array}{ll}1 \times 10^{-4} \\
2.5 \times 10^{-5}\end{array}$ & 5.6 & 1500 & 1.0 & $-0.55_{-0.8}^{-0.2}$ & $83_{50}^{90}$ & $77 \frac{100}{60}$ & 0.08 \\
\hline MWC 297 & 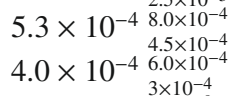 & $\begin{array}{l}5.0 \\
200_{300}^{100}\end{array}$ & 1800 & 1.2 & $-0.77_{-0.9}^{-0.6}$ & $5_{0}^{10}$ & $\begin{array}{l}28.5_{25}^{30} \\
300_{400}^{200}\end{array}$ & 0.19 \\
\hline $\mathrm{LKH} \alpha 215$ & $6.0 \times 10^{-8} 9 \times 10^{-8}$ & 3.5 & & & $-1.4_{-1.8}^{-1.2}$ & $30 \underset{0}{50}$ & $9.2_{-}^{20}$ & 0.1 \\
\hline $\mathrm{Z} \mathrm{CMa}$ & $7.0 \times 10^{-4} \begin{array}{r}1.4 \times 10^{-3} \\
3.5 \times 10^{-4}\end{array}$ & 7.5 & 1500 & 1.9 & $-0.7_{>-1.0}^{-1.0}$ & $30_{20}^{40}$ & $180_{140}^{250}$ & 0.15 \\
\hline
\end{tabular}

${ }^{1}$ In the case of fitted parameters, we provide in small figures the range of allowed values. These values correspond to numerical errors. See Sect. 5.7 for further discussion of the uncertainties in the disk parameters.

interferometric $1.3 \mathrm{~mm}$ and $2.7 \mathrm{~mm}$ data allow us to constrain the mass of dust and estimate the grain sizes. The dust mass in the disk around $\mathrm{R}$ Mon is found to be $1.4 \times 10^{-4} M_{\odot}$ and grain growth has proceeded to sizes of $\sim 1 \mathrm{~cm}$ in the midplane (see Table 4 and Fuente et al. 2003, 2006). Fuente et al. (2006) and Alonso-Albi et al. (2007) demonstrate the existence of a gaseous, flat disk around R Mon. The existence of large grains in the disk midplane is consistent with this geometry. Large grains produce a decrease in the dust opacity and a more rapid photoevaporation of the external layers of the gaseous disk.

The difference between the single-dish and interferometric fluxes at $1.3 \mathrm{~mm}$ is clearly indicative of the presence of an envelope. Close et al. (1997) and Movsessian et al. (2002) inferred that the nebula illuminated by $\mathrm{R}$ Mon has an hourglass shape, and an inclination angle of $\sim 20^{\circ}$ with respect to the sky plane. The same kind of hourglass morphology is observed in the ${ }^{12} \mathrm{CO} 1 \rightarrow 0$ and $2 \rightarrow 1$ images reported by Fuente et al. (2006). To model the envelope, we used a toroid with the same inclination as observed and shadowed by the inner disk. The available photometry includes Spitzer mid-IR observations with a beam size of $30^{\prime \prime}$ and JCMT observations from Mannings (1994) with a beam size of around $18^{\prime \prime}$. We extended the envelope to cover the Spitzer beam size, using a temperature profile $T(r)=62(r(\mathrm{AU}) / 700)^{-0.5} \mathrm{~K}$ (see Table 6$)$. The mass of dust in the envelope was found to be $0.008 M_{\odot}$. The density profile of the envelope was described by a power law with slope -1.4 . This means that an important fraction of the mass is close to the inner radius of the envelope, which is found to be $700 \mathrm{AU}$. The dust temperature at this radius is $60 \mathrm{~K}$.

\subsection{Z CMa}

$\mathrm{Z} \mathrm{CMa}$ is a binary system with a $\mathrm{HBe}$ star and a FU Orionis companion. The most recent estimate of the stellar masses of $\mathrm{Z} \mathrm{CMa}$ and its companion were $16 M_{\odot}$ and $3 M_{\odot}$, respectively (van den Ancker et al. 2004). The available photometry only provides the combined emission of both components, with the exception of the observations by Koresko et al. (1991) which resolved the two stars. The optical observations shown in Fig. 2 should be considered to be a combination of both components. We fitted the optical observations by assuming that the HBe star contributes approximately $30 \%$ of the total flux (van den Ancker et al. 2004).

The SED of Z CMa is shown in Fig. 2. The shape from near to far IR is flat with an abrupt slope in the emission beyond $100-200 \mu \mathrm{m}$. These two characteristics indicate that this is a massive, compact object. Obviously, the disk properties are difficult to derive and the existence of two circumstellar disks, one around each star, and/or a circumbinary disk cannot be discarded. We attempted to reproduce the NIR, mid-IR and FIR SED by assuming one single disk around the HBe star. We were unable to fit the IR SED by assuming a value of $16 M_{\odot}$ for the stellar mass, but we obtained a reasonably good fit by assuming a stellar mass of $12 M_{\odot}$ which is still consistent with being a Be star. On the basis of mainly our interferometric mm observations, we derive a mass of $\sim 7.0 \times 10^{-4} M_{\odot}$ for the circumstellar dust. The $1.3 \mathrm{~mm} / 2.7 \mathrm{~mm}$ spectral index was fitted most accurately with grains of maximum size $\sim 1 \mathrm{~cm}$. 

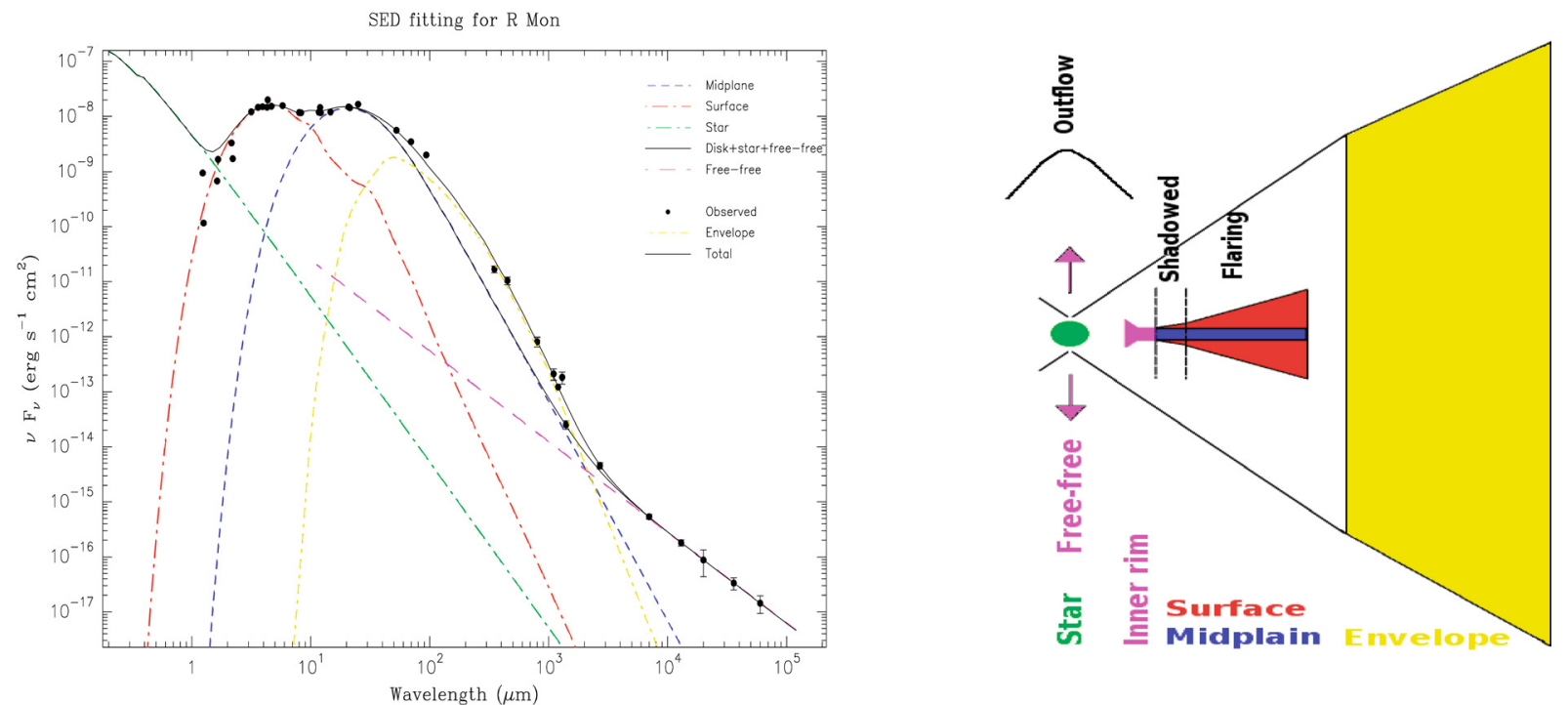

Fig. 1. Left: observed SED and model predictions for R Mon. Right: sketch of our model. The different emission components that contribute to the SED as predicted by our model are drawn in different colors. The emission of the star itself is drawn in green. The disk emission is separated into three components: inner rim emission (magenta), surface layer emission (red), and the midplane emission (blue). Note that the disk around R Mon is fitted without the presence of an inner rim. The emission from the envelope is drawn in yellow. The free-free emission is shown in magenta. The disk emission and the disk+envelope emission appear as two continuous, black lines.

The envelope around $\mathrm{Z} \mathrm{CMa}$ is the most massive and compact in our sample (see Fig. 2 and Table 6), which may be interpreted as proof of the youth of this object. It is well fitted by a shadowed toroid of $r_{\text {in }}=2000 \mathrm{AU}, r_{\text {out }}=5000 \mathrm{AU}$, and an inclination angle of $30^{\circ}$. The abrupt slope in the sub-mm region implies that the envelope cannot be extended beyond an outer radius of $5000 \mathrm{AU}$. For this source, some measurements derived for different beams of fluxes at $450 \mu \mathrm{m}, 850 \mu \mathrm{m}$, and $1.3 \mathrm{~mm}$ are available (Dent et al. 1998; Sandell \& Weintraub 2001). By fitting the fluxes observed with different beams, we inferred a density slope of -0.6 for the envelope, a value close to the density slope of -0.7 found in the disk.

The derived extinction from the envelope model (Table A.8) is 0 since the inclination of the toroid is small, $30^{\circ}$, close to a pole-on view, and the star itself is visible through the conical cavity excavated by the outflow. However, by increasing the inclination to $50^{\circ}$, the extinction increases to a value a factor of 10 higher than the observed value. An outer cold, spherical envelope could possibly account more accurately for the observed extinction.

\subsection{MWC 297}

At a distance of only $250 \mathrm{pc}$, MWC 297 is the closest star in our sample. It is highly reddened with a $B-V$ color of 2.0 , with no known binary companion. At optical wavelengths, a value of $R_{\mathrm{v}}=3.5$ accounted for the extinction better than the value of $R_{\mathrm{v}}=3.1$. At $2 \mu \mathrm{m}$, the SED is almost flat with a small prominence that could be interpreted as an inner rim (see Fig. 3). However, this feature is unclear. We obtained reasonably good fits by using a face-on disk $\left(i \sim 5^{\circ}\right)$ model with an inner rim, or a highly inclined disk $\left(i \sim 80^{\circ}\right)$ without an inner rim. On the basis of IR interferometric observations, Acke et al. (2008) proposed the existence of a low inclination disk ( $i$ less than $40^{\circ}$ ) with dust closer to the star than the dust sublimation radius. We selected the low-inclination solution to be consistent with previous nearIR interferometric measurements by Acke et al. (2008). Since
Table 5. Grain properties in the disks ${ }^{1}$.

\begin{tabular}{lll}
\hline \hline Name & $\begin{array}{l}\text { Midplane } \\
\text { grains }\end{array}$ & $\begin{array}{l}\text { Surface } \\
\text { grains }\end{array}$ \\
\hline MWC 137 & SM $a_{\max }=1 \mathrm{~cm}$ & SM $a_{\max }=1 \mu \mathrm{m}$ \\
R Mon & SM $a_{\max }=1 \mathrm{~cm}$ & $\begin{array}{l}86 \% \text { graphite } 100 \% \\
50 \%\end{array} a_{\max }=1 \mu \mathrm{m}$ \\
MWC 1080 & SM $a_{\max }=1 \mathrm{~cm}$ & SM $a_{\max }=100 \mu \mathrm{m}$ \\
MWC 297 & SM $a_{\max }=1 \mathrm{~cm}$ & SM $a_{\max }=1 \mu \mathrm{m}$ \\
LKHa 215 & SM $a_{\max }=1 \mathrm{~cm}$ & SM $a_{\max }=100 \mu \mathrm{m}$ \\
Z CMa & SM $a_{\max }=1 \mathrm{~cm}$ & $60 \%$ graphite $100 \% a_{\max }=1 \mu \mathrm{m}$ \\
\hline
\end{tabular}

1 The index of the power-law grain-size distribution is fixed $(p=$ 3.5). As a first step, we assumed the standard mixture (SM: $86 \%$ silicates/14\% graphite) in the midplane and surface layers in all the sources. In the cases of $\mathrm{R}$ Mon and $\mathrm{Z} \mathrm{CMa}$, we needed to change the dust composition in the surface to fit the SED. The range of allowed values are indicated (small figures) in the Table. See Sect. 5.7 for a detailed discussion of the uncertainties.

our model is based on hydrostatic equilibrium, it cannot account for the existence of dust closer to the star than the sublimation radius. In our model, we allow the rim temperature $\left(T_{\text {rim }}\right)$ to vary between $1500 \mathrm{~K}$ (silicates sublimation temperature) and $2000 \mathrm{~K}$ (graphite sublimation temperature) and the inner radius is determined by $T_{\text {rim. }}$. The fact that the flux decreases at wavelengths longer than $10 \mu \mathrm{m}$ implies that the circumstellar disk should be small. The most consistent fit is obtained with $T_{\text {rim }} \sim 1800 \mathrm{~K}$, an outer radius of only $\sim 28 \mathrm{AU}$, a density slope of $\sim-0.77$, and an inclination angle of $5^{\circ} \pm 5^{\circ}$. This small disk is optically thick at $1.3 \mathrm{~mm}$ and, consequently, the dust mass and grain properties are not determined (see Table 5). We assume $a_{\max }=1 \mathrm{~cm}$ in the midplane.

The slope of the SED at $\mathrm{mm}$ and sub-mm wavelengths is inconsistent with that expected from an envelope with standard interstellar-medium (ISM) grains. The $1.3 \mathrm{~mm} / 2.7 \mathrm{~mm}$ spectral 
Table 6. Envelope modeling results.

\begin{tabular}{llllllll}
\hline \hline Name & $\begin{array}{l}\text { Dust mass } \\
\left(M_{\odot}\right)\end{array}$ & $\begin{array}{l}\text { Inner radius } \\
(\mathrm{AU})\end{array}$ & $\begin{array}{l}\text { Outer radius } \\
(\mathrm{AU})\end{array}$ & $\begin{array}{l}\text { Density } \\
\text { slope }\end{array}$ & $\begin{array}{l}\text { Inner temperature } \\
(\mathrm{K})\end{array}$ & $\begin{array}{l}\text { Geometry } \\
\text { Inclination } \\
(\text { degrees })\end{array}$ \\
\hline MWC 137 & 0.005 & 5000.0 & 11700.0 & -1.0 & 61 & Sphere & \\
R Mon & 0.008 & 700.0 & 12000.0 & -1.4 & 62 & Toroid $^{1}$ & 70.0 \\
MWC 1080 & 0.025 & 6000.0 & 12000.0 & -1.0 & 44 & Toroid $^{1}$ & 80.0 \\
Z CMa & 0.05 & 2000.0 & 5000.0 & -0.6 & 42 & Toroid $^{1}$ & 30.0 \\
LKH $\alpha 215$ & 0.0015 & 3500.0 & 7200.0 & 0.0 & 57 & Sphere & \\
\hline
\end{tabular}

${ }^{1}$ The dust temperature in the envelope is assumed to be described by a power law, $T(r)=T_{\text {in }}\left(r / r_{\text {in }}\right)^{-0.5}$. See Sects. 4 and 5 for a more detailed discussion.
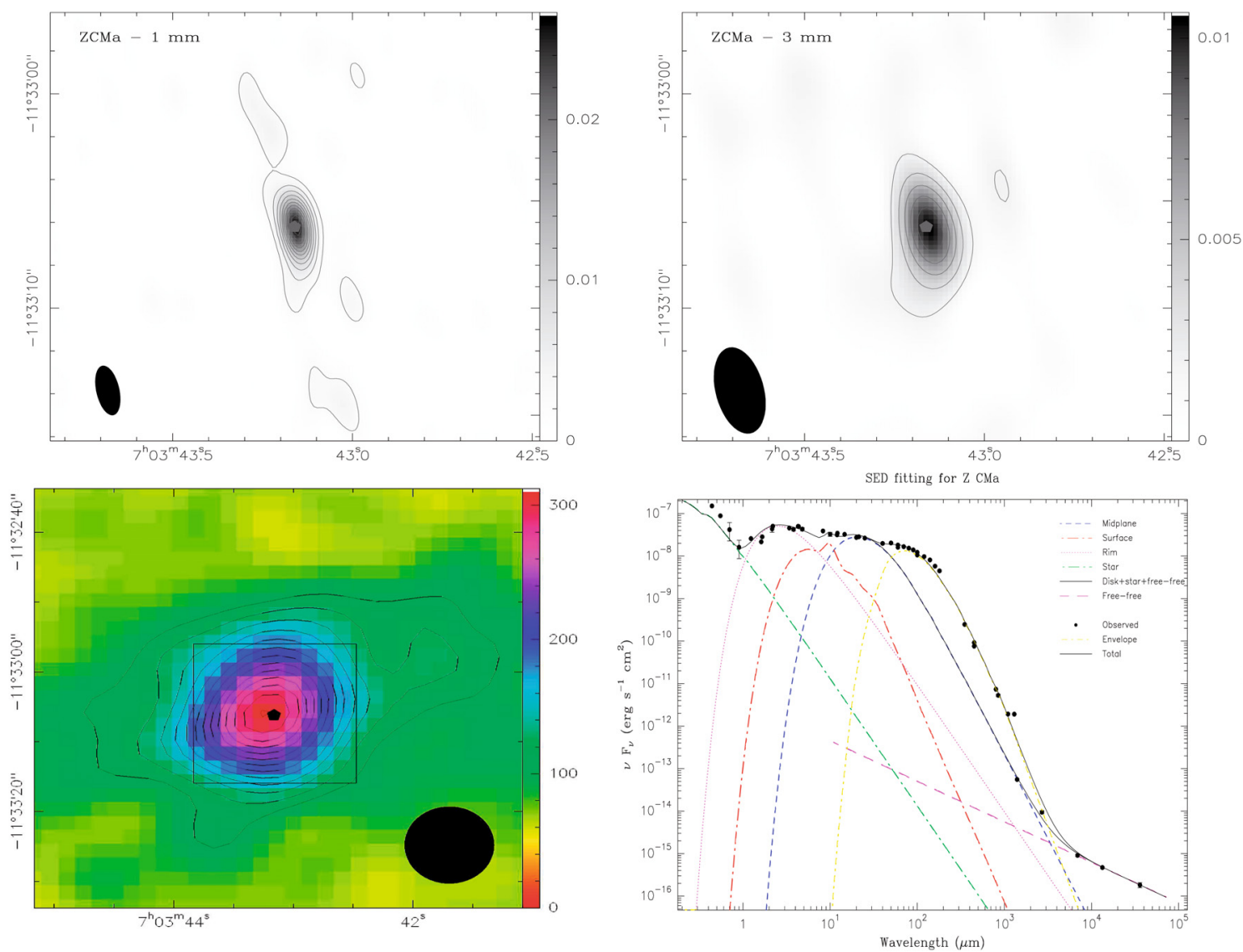

Fig. 2. Up: Z CMa maps obtained with PdBI. Contours in the $1.3 \mathrm{~mm}$ image are represented in steps of $0.77 \mathrm{mJy} / \mathrm{beam}$, starting from $2.31 \mathrm{mJy} / \mathrm{beam}$. In the $2.6 \mathrm{~mm}$ image, the contours are from $1.8 \mathrm{mJy} / \mathrm{beam}$, in steps of $0.6 \mathrm{mJy} / \mathrm{beam}$. Down-Left: Z CMa map at $1.3 \mathrm{~mm}$ obtained with the $30 \mathrm{~m}$ bolometer. Contours are represented in steps of $20 \mathrm{mJy} /$ beam starting from $50 \mathrm{mJy} / \mathrm{beam}$ ( $3 \sigma$ level). Maximum intensity is 293 mJy. The rectangle indicates the 20 arcsec side region shown in the upper panels. Down-Right: the observed SED and our model predictions for Z CMa. The different emission components are drawn following the color code described in Fig. 1.

index is also too low to be adjusted by thermal dust emission. The problem could be the different beam sizes of the interferometric observations at $1.3 \mathrm{~mm}$ and $2.7 \mathrm{~mm}$. In the case that the emission at $1.3 \mathrm{~mm}$ and $2.7 \mathrm{~mm}$ is more extended than the beams, the measured $1.3 \mathrm{~mm} / 2.7 \mathrm{~mm}$ spectral index would be affected by the different angular resolutions. To confirm this possibility, we compared our $1.3 \mathrm{~mm}$ flux with the previous measurement carried out with the SMA providing a beam of $\sim 3^{\prime \prime}$ (Manoj et al. 2007). Our flux 58\% of the flux values measured by Manoj et al. (2007), indicating that our $1.3 \mathrm{~mm}$ observations resolve the $1.3 \mathrm{~mm}$ emission. Thus, we adopted the flux measured by Manoj et al. (2007) to derive the $1.3 \mathrm{~mm} / 2.7 \mathrm{~mm}$ spectral index. Even using this value, the derived spectral index was too low to be due to the ISM grains expected in the envelope. The spectral index was, however, more similar to that found in circumstellar disks, where grain growth occurred. This prompted us to consider the existence of a second disk component towards MWC 297. This second disk could be due to a binary companion, or a ring around the main disk. Since no binary companion was detected close to this star (at a distance of $\sim 0.7^{\prime \prime}$ or $180 \mathrm{AU}$ ), the second option is more likely. We found good agreement with observations by assuming a ring of large grains $(\sim 1 \mathrm{~cm})$, a dust mass of $4 \times 10^{-4} M_{\odot}$, an inner and outer radius of $200 \mathrm{AU}$ and 300 AU respectively, and a temperature profile $T(r)=125(r(\mathrm{AU}) / 200)^{-0.5} \mathrm{~K}$.

The JCMT map (bottom-left panel in Fig. 3) indicates that, in addition to the small disk and ring, there is a more extended 

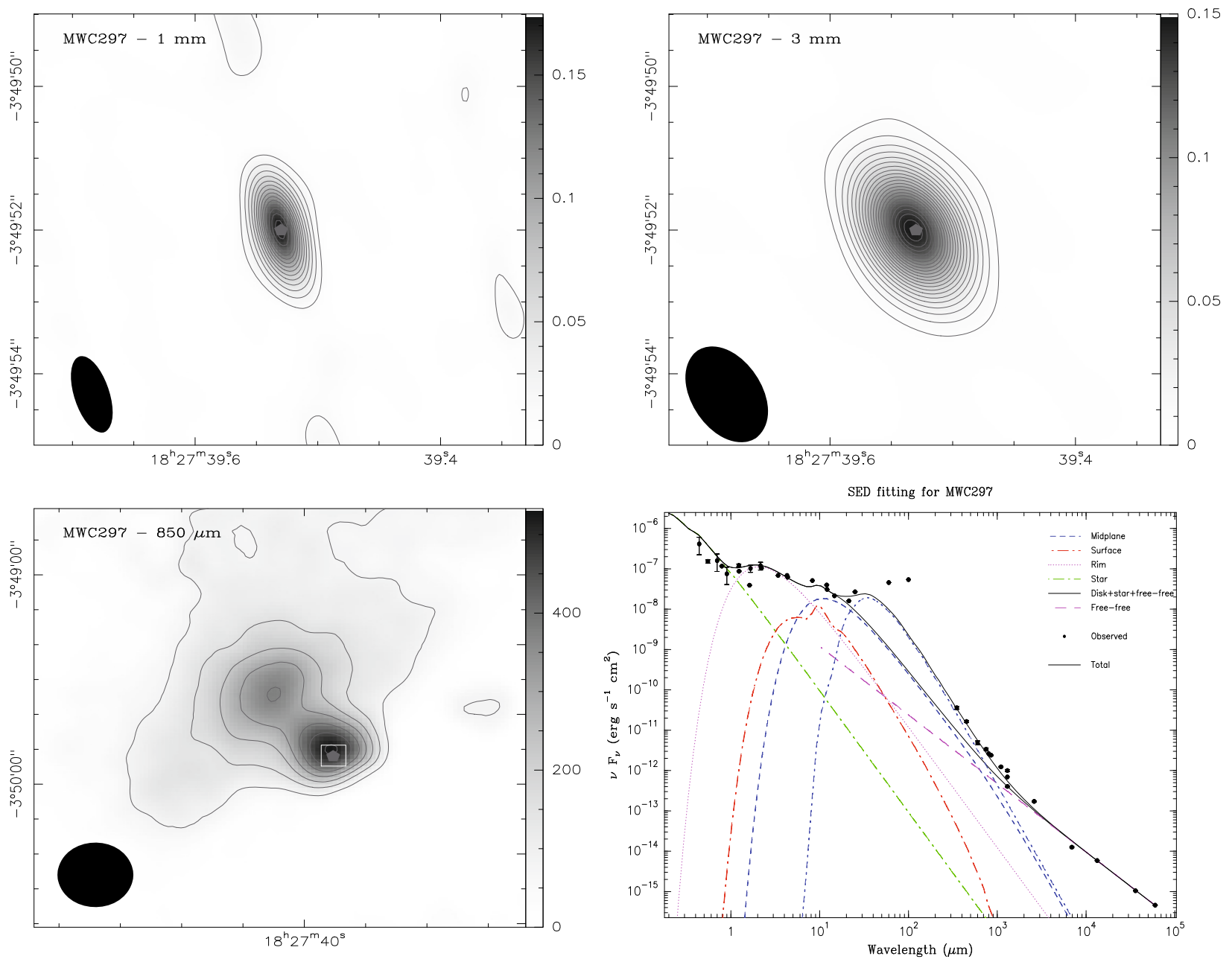

Fig. 3. Up: MWC 297 maps obtained with PdBI. Contours in the $1.3 \mathrm{~mm}$ image are represented in steps of $4 \mathrm{mJy} / \mathrm{beam}$, starting from $12 \mathrm{mJy} / \mathrm{beam}$. In the $2.6 \mathrm{~mm}$ image the contours are from $6.6 \mathrm{mJy} /$ beam, in steps of $2.2 \mathrm{mJy} /$ beam. Down-Left: MWC 297 map at $850 \mu \mathrm{m}$ obtained with JCMT. Contours are represented in steps of $75 \mathrm{mJy} /$ beam, starting from $75 \mathrm{mJy} /$ beam. The small rectangle marks the 6 arcsec side region shown in the upper panels. Down-Right: the observed SED and our model predictions for MWC 297. Note that in this case there is no envelope but two disk components.

envelope surrounding this object. Nebulous emission is also observed in the MIPS $24 \mu \mathrm{m}$ image, suggesting that the star is still embedded in the molecular cloud. However, most of the emission at millimeter wavelengths $(92 \%)$ indicates a small region $\left(\sim 3^{\prime \prime}\right)$ around the star (see Appendix A.7). This is consistent with our two-disk-component fit.

\subsection{MWC 1080}

MWC 1080 was classified as a B0 star. Its distance estimates ranged from $1000 \mathrm{pc}$ (Eisner et al. 2004; Fuente et al. 2003) to $2100 \mathrm{pc}$ (Acke et al. 2005). We adopted the distance of $1000 \mathrm{pc}$, since it is far more consistent with the observed stellar emission after the extinction correction.

The SED towards MWC 1080 has a clear bump at $2 \mu \mathrm{m}$, indicating the presence of a rim with $T_{\mathrm{d}} \sim 1500 \mathrm{~K}$ (see Fig. 4). The dust temperature in the inner rim was determined from the peak at $2 \mu \mathrm{m}$. The mid-IR part of the SED was well fitted with a disk of $r_{\text {out }} \sim 77 \mathrm{AU}$ and an inclination angle of $\sim 80^{\circ}$. This inclination angle is totally consistent with MWC 1080 being an eclipsing binary star, for which the amplitude in the variation of the apparent magnitude is wide (Grankin et al. 1992). The dust mass that better accounts for the $1.3 \mathrm{~mm}$ interferometric flux is found to be $5 \times 10^{-5} M_{\odot}$. The maximum grain size is not determined since we have not detected the disk at $2.7 \mathrm{~mm}$. We obtain a good fit for grains of $\sim 1 \mathrm{~cm}$ in the midplane.

The existence of an envelope is certain since the observed $1.3 \mathrm{~mm}$ interferometric flux is a small fraction $(1.3 \%)$ of the total flux measured with the JCMT. The interferometric 1.3 and $2.7 \mathrm{~mm}$ images also reveal the existence of several clumps surrounding the circumstellar disk (see Fig. 4 and Fuente et al. 2003). On the basis of the morphology observed in our interferometric images, we used a toroid with an inner radius of $6000 \mathrm{AU}$ (6" at the distance of MWC 1080) to fit the envelope. The best fit solution for the envelope is a non-shadowed toroid of an outer radius of $12000 \mathrm{AU}\left(12^{\prime \prime}\right)$. Our model fits all the SED points except the IRAS flux at $100 \mu \mathrm{m}$. This is unsurprising since the wide IRAS beam at $100 \mu \mathrm{m}\left(\sim 2^{\prime}\right)$ probably detects emission from the foreground molecular cloud.

The extinction estimate from the $B-V$ color index is $40 \%$ higher than that predicted by our envelope model. This is also consistent with the scheme of the star+disk+toroid system immersed in a lower-density molecular cloud. 

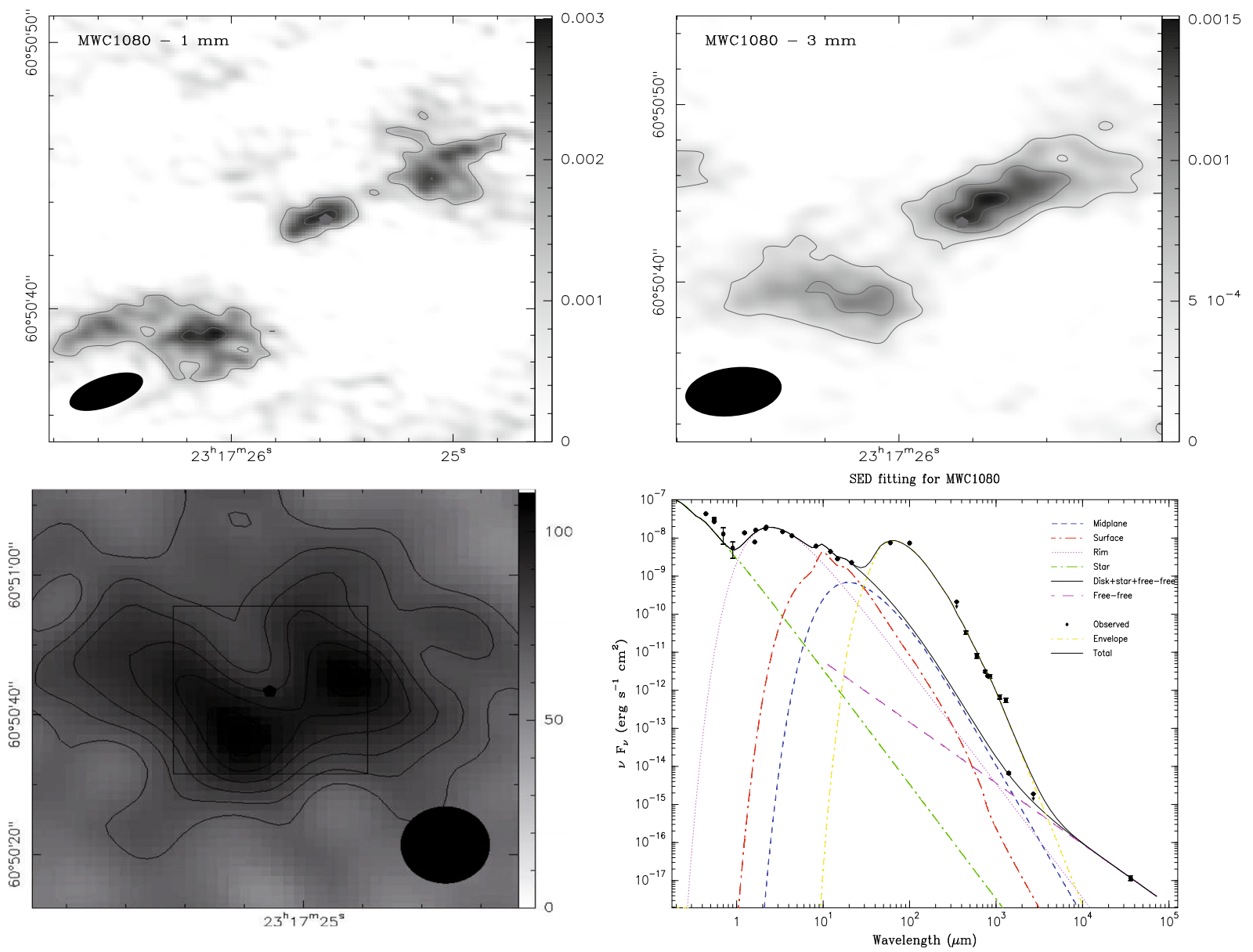

Fig. 4. Up: MWC 1080 maps at $1.3 \mathrm{~mm}$ and $2.6 \mathrm{~mm}$ obtained with PdBI. Contours in the $1.3 \mathrm{~mm}$ image are represented in steps of $0.45 \mathrm{mJy} / \mathrm{beam}$, starting from $1.35 \mathrm{mJy} / \mathrm{beam}$. In the $2.6 \mathrm{~mm}$ image, the contours are from $0.45 \mathrm{mJy} / \mathrm{beam}$, in steps of $0.15 \mathrm{mJy} / \mathrm{beam}$. Down-left: MWC $1080 \mathrm{map}$ at $1 \mathrm{~mm}$ obtained with the $30 \mathrm{~m}$ bolometer. Contours are represented in steps of $10 \mathrm{mJy} / \mathrm{beam}$ starting from $40 \mathrm{mJy} / \mathrm{beam}(3 \sigma$ level). Maximum intensity is $95 \mathrm{mJy}$. The 26 arcsec side square indicates the region shown in the PdBI $2.6 \mathrm{~mm}$ map. Down-right: the observed SED and our model predictions for MWC 1080.

\subsection{MWC 137}

The SED of MWC 137 does not exhibit any evidence of disk emission at $\mathrm{mm}$ wavelengths. All the mm-cm fluxes can be fitted by a single component of spectral index $\alpha=+0.76 \pm 0.01$. This spectral index is consistent with that expected in the freefree emission arising in the stellar wind. Although a value of $\alpha=+0.6$ is expected for an ionized isotropic wind, small deviations of this value can be explained by a different geometry or a partially ionized wind. However, some excess is visible at IR wavelengths, which is suggestive of the presence of a small disk. Fuente et al. (2003) derived an upper limit to the dust mass of $7 \times 10^{-5} M_{\odot}$ assuming that the disk is optically thin at $1.3 \mathrm{~mm}$, the free-free emission spectral index is +0.6 , and standard values for the dust opacity and mean dust temperature (see Sect. 6). However, this value is quite uncertain since the disk is probably optically thick and the grain emissivity is unknown. We fitted the NIR part of the SED and obtained the best-fit solution (consistent with our mm observations) for a small disk of $r_{\text {out }} \sim 18 \mathrm{AU}$ and a dust mass of $10^{-5} M_{\odot}$. The absence of a large disk ensure that the envelope is prominent in the SED. The envelope is modeled as an sphere extended between 5000 and $11700 \mathrm{AU}$, with a dust mass of $0.005 M_{\odot}$ (see Fig. 5).

\section{6. $L K H \alpha 215$}

We did not detected this source at $\mathrm{mm}$ and $\mathrm{cm}$ wavelengths. This implies an upper limit to the dust mass of $9.0 \times 10^{-5} M_{\odot}$, assuming optically thin emission and standard values of the dust temperature and emissivity. We modeled the entire SED to improve the upper limit to the mass of dust and to determine the envelope component (see Fig. 6). Our best fit, still although uncertain, solution is obtained for a disk with an outer radius of $\sim 10 \mathrm{AU}$ and a mass $\sim 6 \times 10^{-8} M_{\odot}$. The envelope is described well by a dust mass of about $\sim 0.0015 M_{\odot}$, located between $\sim 3500 \mathrm{AU}$ and $\sim 7200 \mathrm{AU}$.

\subsection{Uncertainties in the disk mass and maximum grain size}

The dust mass and the grain properties determined by fitting the SED are affected by important uncertainties. The most important assumption is that the dust emission is optically thin at $\mathrm{mm}$ wavelengths. This is the case for typical circumstellar disks of $r_{\text {out }} \sim 100 \mathrm{AU}$ and dust mass $<10^{-3} M_{\odot}$. Only in the case of small disks, the $1.3 \mathrm{~mm}$ emission could become optically thick.

We discuss R Mon as a representative case of an optically thin disk. Our best fit solution is for a disk with $r_{\text {out }}=150 \mathrm{AU}$ and a dust mass of $1.4 \times 10^{-4} M_{\odot}$. This disk is optically thin 
at $1.3 \mathrm{~mm}$. A disk of this size needs to have a dust mass of $\sim 0.01 M_{\odot}$, i.e. a factor of 100 higher than our estimate, to become optically thick $\left(\tau_{1.3 \mathrm{~mm}} \sim 1\right)$. But in this case, the flux at $1.3 \mathrm{~mm}$ would be more than one order of magnitude higher than the measured flux (see Fig. 7). In the optically thick case, the flux at $1.3 \mathrm{~mm}$ depends only on the area of the disk projected on the plane of the sky and the dust temperature across the disk surface, $F_{1.3 \mathrm{~mm}} \sim B_{\lambda}\left(T_{\mathrm{s}}\right) \pi r_{\text {out }}^{2} \cos (i)$. The only way of reconciling the predicted mm flux with observations is by reducing the size of the disk and/or changing the disk inclination. But in that case we obtain a deficient emission in the NIR and mid-IR range as it is illustrated in Fig. 7. The same argument is valid for Z CMa.

A different case is the small disk surrounding MWC 297. In this case, the disk is optically thick and the derived mass is only a lower limit to the true disk mass. However, it is unreasonable to propose that the dust mass in the small and, probably, evolved disk around MWC 297 is larger than that within the less evolved disk around R Mon. One would expect that the mass of dust and gas in the disk decreases because of photoevaporation (see Sect. 6). Thus, our mass should be accurate to within an order of magnitude.

The SED towards MWC 1080 is well described by a partially optically thin disk as shown in Table 4. But it can also be reasonably well reproduced by an optically thick disk with $r_{\text {out }}=50 \mathrm{AU}$ and a dust mass $\geq 10^{-3} M_{\odot}$. Following the same argument as for MWC 297, it is unreasonable to assume that the dust mass in this small disk is one order of magnitude larger than in the less evolved disk around R Mon and similar to those found in TTs. Thus, we keep the optically thin solution, although we are aware of the uncertainty in our analysis.

In the case of optically thin disks, we can learn about the grain properties from the $1.3 \mathrm{~mm} / 2.7 \mathrm{~mm}$ spectral index. In the Rayleigh-Jeans region of the spectrum, the dust emission is $\alpha v^{(2+\beta)}$ where $\beta$ is the opacity index that can be accurately derived by fitting the observed SED. The value of $\beta$ in the submm$\mathrm{mm}$ range is an excellent indicator of the grain size distribution (Draine 2006). The grain size distribution is usually taken to be, $n(a)=n_{0} a^{-p}$ for $a<a_{\max }$, where $n_{0}$ is a normalization factor, $p$ is a parameter in the range 2.5-3.5, and $a_{\max }$ is the maximum grain size. The value of $\beta$ is between 1 and 2 for small grains (sub-micron-sized grains) but becomes lower than 1 when $a_{\max }$ is a few $\mathrm{mm}$. To have a value of $\beta \leq 0.5$, we need grains with $a_{\max } \sim 1 \mathrm{~cm}$. This is the case for R Mon and Z CMa. However, there is some degeneracy between the index of the grain size distribution, $\mathrm{p}$, and the value of the maximum grain size, $a_{\max }$. A slope $p=2.5$ means that a larger number of grains are closer to the maximum grain size than for a more typical slope of 3.5. By assuming $p=2.5$ we would reach the same values of $\beta$ with grains slightly smaller. Even in this case, we would require grains with radii of nearly $1 \mathrm{~cm}$, i.e. significant grain growth must have occurred in the disk midplane to have the values of $\beta<0.5$ that we obtain in our sources. We cannot determine grain sizes larger than a few $\mathrm{cm}$ on the basis of $\mathrm{mm}$ observations. The $1.3 \mathrm{~mm} / 2.7 \mathrm{~mm}$ grain opacity index tends asymptotically to 0 for $a_{\max }>a$ few cm. In the case of optically thick disks, we cannot determine the grain size from the SED. We assumed $a_{\max } \sim 1 \mathrm{~cm}$ in the midplane for MWC 1080, MWC 297, MWC 137, and $\mathrm{LkH} \alpha 215$, since grain growth is expected to have proceeded in these more evolved disks.

The dust composition in the midplane cannot be inferred from the SED. For this reason, we assumed the standard mixture in all of our sources (see Table 5). The silicate feature at $\sim 9.8 \mu \mathrm{m}$ provides, however, some information about the dust composition in the surface layer. In all of our sources, the

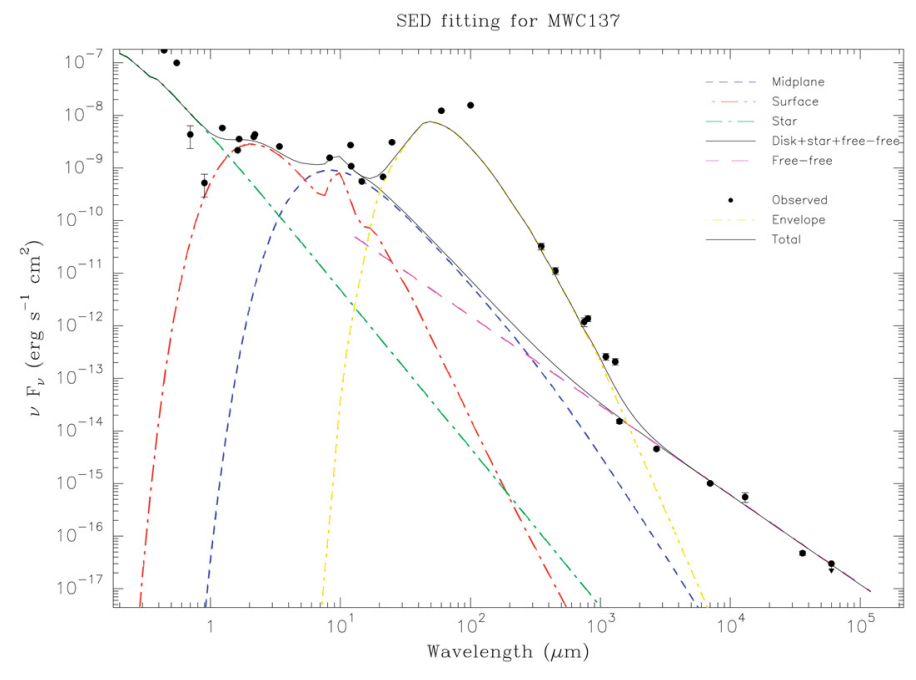

Fig. 5. The observed SED and our model predictions for MWC 137.

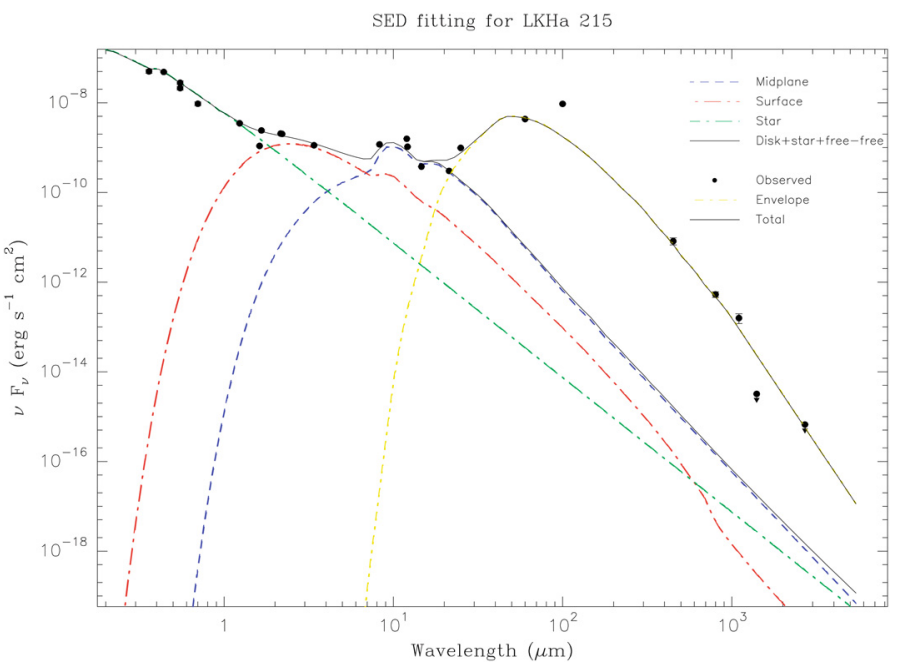

Fig. 6. The observed SED and our model predictions for LKH $\alpha 215$.

silicate feature is weak or absent. The absence of this feature does not imply the absence of silicate grains but the lack of silicate grains at a temperature of $\sim 800 \mathrm{~K}$. The silicate grains could be either too far from the central source and thus not heated to temperatures sufficiently high to allow emission in the mid-IR, or these grains could be too large to be heated efficiently. To investigate the second possibility, we tried to fit SEDs by assuming a standard dust composition and varying the $a_{\max }$ in the surface between $0.1 \mu \mathrm{m}$ up to $100 \mu \mathrm{m}$. We were able to reproduce the disks around MWC 137, MWC 1080, MWC 297, and LkH $\alpha 215$ (see Table 5), but failed to account for R Mon and Z CMa. In these cases, we needed to vary the dust composition to fit the mid-IR part of the SED. The most extreme case was R Mon in which the best fit solution was found for $86 \%$ of graphite in the surface layer (see Fig. 7). This result is, of course, limited by the simplicity of our model. We can have a different geometry from that expected in a thermal equilibrium disk. Alternatively, the dust composition and/or grain growth could vary as a function of the distance from the star. Determining the dust composition of the disk surface is, however, beyond the scope of this Paper, 
and, in addition, does not affect our results because the disk mass depends only on the dust composition and grain size in the midplane. Therefore, we do not discuss this point further.

The dust composition and maximum grain size in the midplane determine the value of dust emissivity at $1.3 \mathrm{~mm}, \kappa_{1.3 \mathrm{~mm}}$, and consequently has an influence on the derived dust mass. Assuming a standard dust mixture and $a_{\max }=1 \mathrm{~cm}, \kappa_{1.3 \mathrm{~mm}}=$ $0.88 \mathrm{~g}^{-1} \mathrm{~cm}^{2}$. This value is close to the canonical value of $1 \mathrm{~g}^{-1} \mathrm{~cm}^{2}$. For reasonable values of $a_{\max }$ in disks, i.e. of between $1 \mathrm{~mm}$ and $1 \mathrm{~cm}$, the value of $\kappa_{1.3 \mathrm{~mm}}$ varies by a factor of 2-3 from the canonical value. This is the uncertainty in the disk mass estimate due to the uncertainty in the grain size in the midplane.

Summarizing we conclude that our disk-mass estimates are accurate to within a factor of 2-3 in the optically thin disks around $\mathrm{R}$ Mon and $\mathrm{Z} \mathrm{CMa}$, and to within an order of magnitude for the optically thick disks around MWC 1080 and MWC 297.

NIR interferometric observations by Eisner et al. (2007) and Acke et al. (2008) revealed the existence of hot dust in a region at $<1$ AU from the star towards MWC 297 and MWC 1080. We are aware that our model is too simple to determine the properties of the disk in a few AU around the star. First of all, in our model, the inner radius of the disk is determined by the dust sublimation temperature that we assume to be between $1500 \mathrm{~K}$ (silicate sublimation temperature) and $2000 \mathrm{~K}$ (graphite sublimation temperature). We do not consider the possibility of the existence of dust closer to the star, as proposed by Eisner et al. (2007) and Acke et al. (2008). Secondly, this extremely hot dust (at less than $1 \mathrm{AU}$ ) would emit radiation at $<2 \mu \mathrm{m}$ and the SED at these wavelengths is dominated by the emission of the stellar photosphere. Our model and method (SED fitting) are indeed inadequate to determine the properties of the innermost regions of the disk ( $r \leq a$ few AU). NIR interferometric observations capable of separating the star from the disk are required to probe this region.

We conclude that the SED fitting technique is sufficiently robust to reproduce the global parameters (dust mass, grain growth) of the outer disks. We emphasize that our analysis describes reasonably well the SED for a wide range of wavelengths $(3 \mu \mathrm{m}-3.6 \mathrm{~cm})$ with a consistent set of star, disk, and envelope parameters. Our results are also consistent with the morphology observed in the millimeter and mid-infrared images.

\section{Discussion}

Our $\mathrm{cm}$ and $\mathrm{mm}$ interferometric observations have provided valuable insight into the structure of the circumstellar matter around $\mathrm{HBe}$ stars. The first result is that $\mathrm{HBe}$ stars are usually surrounded by a massive envelope. The mass of the envelope is always larger than the mass of the disk and dominates the emission at FIR and millimeter wavelengths. Extended emission arising in the envelope is also observed in the MIPS images at $24 \mu \mathrm{m}$, which shows that the envelope emission significantly contributes to the SED even at mid-IR wavelengths. The existence of a nebula was also inferred for Spitzer images towards the A0 star VV Ser (Pontoppidan et al. 2007). Our observations corroborate that interferometric observations are required to derive the properties of the disks around these massive stars, and a disk+envelope model is essential when interpreting the SED.

We have different types of disks in our sample. Towards $\mathrm{Z}$ CMa and $\mathrm{R}$ Mon, we detected disks with sizes of $r_{\text {out }}>$ $100 \mathrm{AU}$ and dust masses of $10^{-4}-10^{-3} M_{\odot}$. These values do not differ from the estimated sizes of disks around TTs and HAe stars. Both of these disks were detected in the
$\mathrm{CO} J=1 \rightarrow 0$ and $J=2 \rightarrow 1$ rotational lines (Fuente et al. 2006; Alonso-Albi et al. 2009). Z CMa is a controversial case. Its accretion rate of $10^{-3} M_{\odot} \mathrm{yr}^{-1}$ (Hartmann et al. 1989) is one of the highest known around a pre-main sequence object, and $\mathrm{Z}$ CMa is surrounded by a compact, massive envelope and powers an energetic outflow (Poetzel et al. 1989). It is probably the youngest object in our sample, and it probably contains the most massive disk. However, the dust emission could originate in a circumbinary ring and/or a disk associated with the FU Orionis companion.

The circumstellar disk around MWC 1080 is smaller $\left(r_{\text {out }} \sim\right.$ $80 \mathrm{AU})$ and less massive $\left(5 \times 10^{-5} M_{\odot}\right)$ than those around $\mathrm{Z} \mathrm{CMa}$ and R Mon. However, the envelope contains a significant amount of dust, which suggests that the star is still quite young. This disk has not been detected in the $\mathrm{CO} J=1 \rightarrow 0$ and $2 \rightarrow 1$ lines (Alonso-Albi et al., unpublished data).

MWC 297 is a special case because its disk consists of two components. The inner disk has a radius of $28 \mathrm{AU}$, similar to the more evolved sources discussed below, while the outer ring has a radius of $200 \mathrm{AU}$, more similar to the young disks around $\mathrm{R}$ Mon and $\mathrm{Z}$ CMa. The disk has not been detected by its $\mathrm{CO}$ $J=1 \rightarrow 0, J=2 \rightarrow 1$ (Alonso-Albi et al. unpublished data) nor $J=3 \rightarrow 2$ (Acke et al. 2008) rotational line radiation.

Although the mass of dust in the circumstellar disk varies between one source and another $\left(10^{-5}-10^{-3} M_{\odot}\right)$, the $1.3 \mathrm{~mm} / 2.7 \mathrm{~mm}$ spectral index is low $(<2.2)$ in all sources. This $1.3 \mathrm{~mm} / 2.7 \mathrm{~mm}$ spectral index indicates that grain growth has proceeded to sizes of $1 \mathrm{~cm}$ even in the youngest sources of our sample, R Mon and Z CMa. As discussed later, early grain growth has important consequences for the evolution of the dusty disk. The non-detection at $\mathrm{mm}$ wavelengths of the disks around MWC 137 and $\mathrm{LkH} \alpha 215$ also implies the existence of compact $(<20 \mathrm{AU})$ and/or presumably light disks $\left(<0.001 M_{\odot}\right)$, if any, around these stars.

\subsection{Disk masses around Herbig Ae/Be stars}

To investigate the possible dependence of the disk mass on the stellar mass and age, we compiled a list of disks detected at $\mathrm{mm}$ wavelengths $(1.1-2.7 \mathrm{~mm}$ ) around HAeBe stars (see Table A.13). It is important to calculate the disk masses in a uniform way. We calculated all disk masses, including for our own sample, by assuming optically thin emission at mm wavelengths, an average disk temperature that depends on the stellar spectral type (Natta et al. 2000), a dust opacity $\kappa_{1.3 \mathrm{~mm}}=1.0 \mathrm{~cm}^{2} \mathrm{~g}^{-1}$, and a dust spectral index $\beta=1.0$. We subtracted the free-free contribution in the sources previously studied by our team (Fuente et al. 2003, 2006; Alonso-Albi et al. 2008). Then we calculated the disk masses by assuming that all the $1.3 \mathrm{~mm} / 2.7 \mathrm{~mm}$ emission originates in the dusty disk. To estimate the uncertainty in the disk masses derived in this "simple" way, we compared the disk masses for our sample with those previously derived by modeling the full SED in this Paper. The values agree within a factor $2-3$, which suggests that our method is robust to within the statistical uncertainties.

We also calculated the mass and age of all of these stars using the evolutionary tracks of Siess et al. (2000) and the values of luminosities and effective temperatures found in the literature (see Table A.13). In some cases, the luminosities and effective temperatures were modified slightly (within the expected uncertainty) to fit the closest Siess et al. (2000) evolutionary track and the spectral type of each star. Stars with masses higher than $7 M_{\odot}$ do not undergo a pre-main sequence phase and their ages cannot be estimated using the HR diagram. In these cases, we 

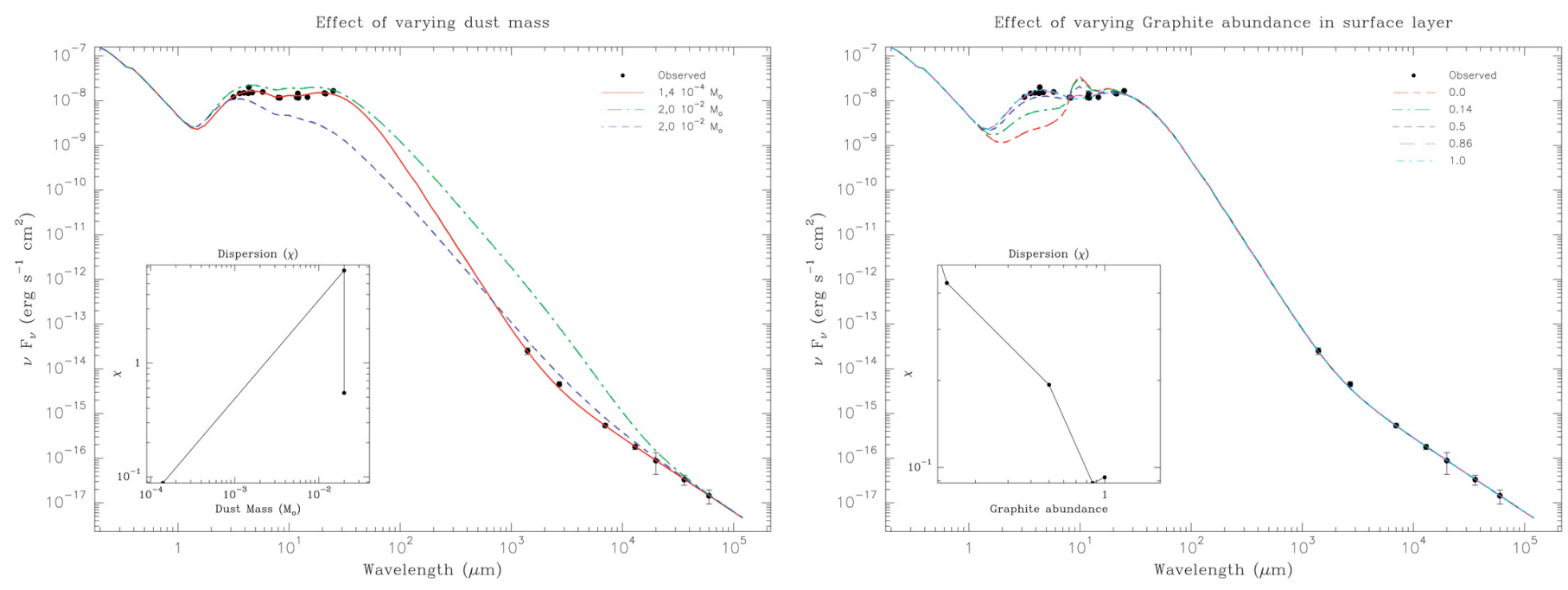

Fig. 7. Left: the SED of R Mon cannot be fitted assuming an optically thick disk. In this case, we obtain a large excess at mm wavelengths (green short-long dashed line). To reduce this excess, we can adjust the other disk parameters, such as the inclination angle or the outer radius, to reduce the effective surface of the disk. But in this case, we obtain a deficient emission at near and mid-IR wavelengths (blue dashed line). The red continuous line shows our optically thin solution. The lower-left chart shows the evolution in the dispersion parameter $(\chi)$. Right: the SED of $\mathrm{R}$ Mon and Z CMa cannot be fitted using a standard grain mixture ( $86 \%$ of astronomical silicate and $14 \%$ of graphite). The pink line shows our best fit for a non-standard mixture with $86 \%$ of graphite. With $100 \%$ of graphite the fit is still excellent, but slightly worse. When the fraction of silicates becomes important (50\% or higher) the model is inconsistent with the observations.
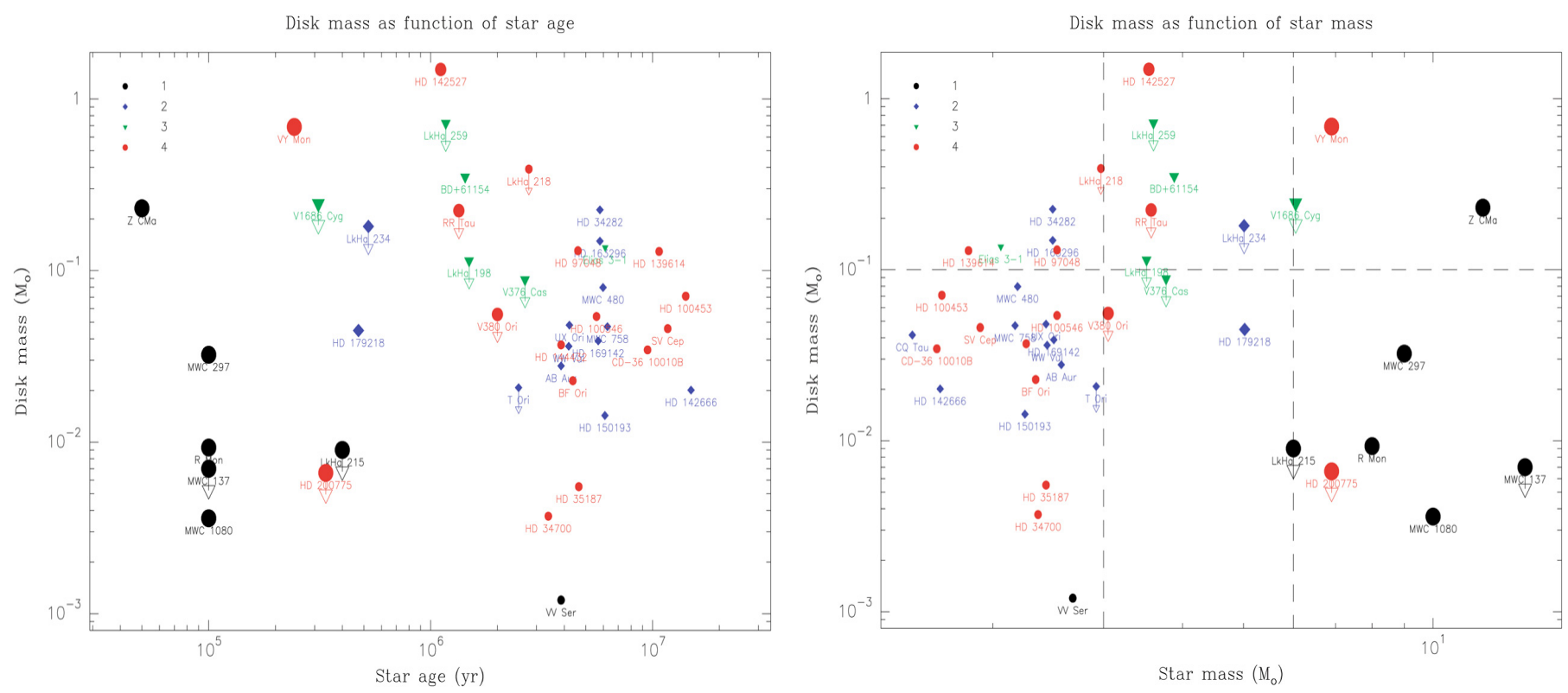

Fig. 8. Compilation of disks masses (gas+dust) as function of stellar age (left) and the stellar mass (right). Our observations with black circles. In blue diamonds we represent the disk masses derived from $1.3 \mathrm{~mm}$ interferometric observations from other authors. With green inverted triangles we represent the disk masses derived from $2.7 \mathrm{~mm}$ interferometric observations. In these stars the disk mass is probably overestimated because of the possible (and not subtracted) contribution of the free-free emission. The red circles correspond to single-dish $1.3 \mathrm{~mm}$ observations. The disk mass in these cases could also be overestimated because of the existence of an envelope.

evaluated the age on the basis of different arguments. For Z CMa, we assumed an age of $0.05 \mathrm{Myr}$ consistent with the time required to form a star of $16 M_{\odot}$ with a mean accretion rate of $3 \times 10^{-4} M_{\odot} \mathrm{yr}^{-1}$. This star could have been even younger if the accretion rate had remained close to $10^{-3} M_{\odot} \mathrm{yr}^{-1}$ throughtout the formation process. MWC 137, MWC 297, MWC 1080, and $\mathrm{R}$ Mon are very likely young main-sequence objects. For these objects, we assumed that the age was lower than 1 Myr based on the post-main sequence tracks of Schaller et al. (1992). For clarity and to differentiate them from other sources in which we have been able to estimate stellar ages, we plot them in the $0.1 \mathrm{Myr}$ slot in the disk mass versus stellar age diagram.

In Fig. 8 (right panel), we present the total disk masses (gas+dust) as a function of the stellar mass assuming a gas/dust ratio of 100 . We can easily distinguish three regions in the plot. Firstly, for stars with masses of $<3 M_{\odot}$, there is a cloud of points 
around a mean value of $\sim 0.04 M_{\odot}$. However, there are a few stars with light disks $\left(<0.01 M_{\odot}\right)$. Secondly, in stars with masses of 3-7 $M_{\odot}$, there is a group of massive disks $\left(>0.1 M_{\odot}\right)$. Most of these disks masses were derived from $2.7 \mathrm{~mm}$ observations and were probably overestimated because of the unsubtracted free-free emission. In the case of HD 142527 and VY Mon, the disk masses were derived from single-dish observations and we cannot discard the contribution of an associated nebula. Thus, these massive disks requires further confirmation. Thirdly, in more massive stars $\left(>7 M_{\odot}\right)$, mainly our entire sample of $\mathrm{HBe}$ stars, the disk masses decrease by a factor 5-10 and become $<0.01 M_{\odot}$. The only exception is $\mathrm{Z} \mathrm{CMa}$, a particularly young object as discussed above. Since it is a binary containing a $16 M_{\odot}$ and $3 M_{\odot}$ stars, the circumstellar disk could be associated with the lower mass component and/or a circumbinary disk. Another interesting case is MWC 297. If we consider only the inner disk, it is located in the region of the "light disks" $\left(<0.01 M_{\odot}\right)$ related to massive stars. If we consider the sum of the two disk components, the disk mass is similar to those of the disks associated with HAe stars.

In Fig. 8 (left panel), we show the disk masses as a function of the stellar age. We can also distinguish the three regions described above. The cloud of points of stars with masses $<3 M_{\odot}$ and disk masses $\sim 0.04 M_{\odot}$ corresponds to stars of ages between a few Myr and 10 Myr. The most massive disks corresponds to stars of masses between 3-7 $M_{\odot}$ and ages of around 1 Myr. The circumstellar disks around stars with masses of $>7 M_{\odot}$ exhibit a large mass dispersion, ranging from $>0.1 M_{\odot}$ to $<0.01 M_{\odot}$, i.e., two orders of magnitude, although mainly at the lower end of this range.

To attempt to understand this behavior, we recall that we only consider pre-main sequence (visible) objects. Thus, there is a lower limit to the age of the star that corresponds to the birthline and depends on the stellar mass. The upper limit to the stellar ages for our disk detections is given by the disk lifetime which probably also depends on stellar mass. For Herbig Ae stars with masses between 1-3 $M_{\odot}$, this period (from the birthline until the disk disperses) corresponds to stellar ages of between a few Myr and 10 Myr. During this phase the disk mass does not vary significantly with stellar age. For stars with masses in the range 3-7 $M_{\odot}$, this phase lasts from $10^{5}$ yr to $\sim 2$ Myr. For the most massive stars, $>7 M_{\odot}$, once the star is visible the disk dispersal is so rapid that instead of having a cloud of points around a mean value, we have a large dispersion of the disk masses ( 2 orders of magnitude), and most of objects in the lower end $\left(M \leq 0.01 M_{\odot}\right)$. This indicates that, although massive disks are found towards young, intermediate-mass protostars (Cesaroni et al. 2005; Schreyer et al. 2006) and possibly Z CMa, after 1 Myr massive stars have already dispersed most of the circumstellar material and are surrounded by disks of masses lower than $0.01 M_{\odot}$.

\subsection{Disk photo-evaporation in massive stars}

Different dynamical processes contribute to the dispersal of gas in circumstellar disks: viscosity, winds and jets, dynamical encounters with nearby stars, and photoevaporation by the central star and external illumination. The relative importance of these processes was discussed by Hollenbach et al. (2000), who concluded that the dominant processes affecting disk evolution are viscosity in the inner regions $(<10 \mathrm{AU})$ and photoevaporation at larger radii. Photoevaporation affects firstly the outer region of the disk, where the gravitational field is lower and the thermal heating can disrupt the disk (see e.g. the review by
Table 7. Dissipation timescale.

\begin{tabular}{llll}
\hline \hline Source & $\begin{array}{l}R_{\mathrm{g}}^{1} \\
(\mathrm{AU})\end{array}$ & $\begin{array}{l}\text { Mass loss rate } \\
\left(M_{\odot} \mathrm{yr}^{-1}\right)\end{array}$ & $\begin{array}{l}\text { Life time }^{2} \\
(\mathrm{yr})\end{array}$ \\
\hline MWC 137 & 124.6 & $8.9 \times 10^{-6}$ & $1.1 \times 10^{5}$ \\
LKH $\alpha$ 215 & 53.4 & $3.4 \times 10^{-6}$ & $2.9 \times 10^{5}$ \\
R Mon & 71.2 & $4.1 \times 10^{-6}$ & $2.4 \times 10^{5}$ \\
Z CMa & 106.8 & $6.1 \times 10^{-6}$ & $1.6 \times 10^{5}$ \\
MWC 297 & 80 & $6.0 \times 10^{-6}$ & $1.7 \times 10^{5}$ \\
MWC 1080 & 89 & $4.6 \times 10^{-6}$ & $2.2 \times 10^{5}$ \\
\hline
\end{tabular}

${ }^{1}$ Gravitational radius calculated following expression (1) by Alexander (2008) $;{ }^{2}$ time required to disperse a $1 M_{\odot}$ disk.

Alexander 2007). We can define a characteristic radius $R_{\mathrm{g}}$ beyond which material evaporates and a gap opens in the disk. As soon as the gap opens, the direct photoevaporation disperses the gaseous disk from the inside to the outside in a few $10^{5} \mathrm{yr}$.

While the gas evolution is outlined above, the dust evolution is more difficult to predict and depends on the maximum grain size (Garaud 2007). Particles are coupled to the gas by means of the frictional drag that depends on grain size. If the particles are far smaller than the mean free path of the gas molecules $\left(\lambda_{\mathrm{mfp}}\right)$, gas and grains are coupled. Assuming typical gas densities, the grains with sizes smaller than $1 \mu \mathrm{m}$ remain coupled to the gas. Thus, if grain growth is ignored, the evolution of the solid particles relates to that of the gas and the dusty disk is rapidly dispersed once the gap is opened. However, if grain growth is taken into account the evolution of the dusty disk is different. Although small particles are blown away with the gas, the large particles remain forming a compact, dusty disk. Alexander \& Armitage (2007) showed that there is a radius beyond which all solids are entrained in the gas. This radius is identified with the region where the maximum grain size is between $1-10 \mathrm{~cm}$.

We propose that the presence of compact, dusty disks around most $\mathrm{HBe}$ stars is the consequence of rapid disk photoevaporation in these massive stars. Since the ionizing flux in $\mathrm{HBe}$ stars is higher than in the cooler HAe and TT stars, the timescale for disk photoevaporation is shorter. In Table 7 we show the characteristic gap radius and the photoevaporation rate for the stars in our sample calculated following Alexander (2008). The massloss rate is about an order of magnitude higher in Be stars than in Ae stars. While VV Ser as an A0 star needs about $9 \mathrm{Myr}$ to dissipate $1 M_{\odot}$ disk mass, HBe stars disperse their gaseous disk on a few $10^{5}$ yr. This time is so short that the star could dissipate the outer part of the disk before becoming visible. As soon as the outer disk is dispersed, the entire gaseous disk is dispersed on a short timescale. This is consistent with the lack of CO detection in the small disks, MWC 1080 and MWC 297, of our sample. One important result of our mm-wave analysis is that grain growth begins early in disk evolution, and grains have grown to sizes of $\sim 1 \mathrm{~cm}$ even before the star becomes visible. Thus, once the gaseous disk is dispersed, a compact, dusty disk formed by large grains remains. An interesting case is MWC 297 in which we find a small, circumstellar disk $\left(r_{\text {out }}=29 \mathrm{AU}\right)$ and a remnant, outer ring with $r_{\text {in }}=200 \mathrm{AU}$ and $r_{\text {out }}=300 \mathrm{AU}$. This second ring could be the consequence of the decrease in gas density outwards from the star centre. The grains entrained in the retreating gas are left behind when the gas density is too low for the drag force to push them away. MWC 297 could be an object in-between the young disks associated with R Mon and Z CMa and the compact, dusty disk surrounding MWC 1080 and presumably, MWC 137 and $\mathrm{LkH} \alpha 215$. 
Additional support for the rapid photoevaporation of disks in HBe stars is found by Berné et al. (2009) on the basis of the PAH and small grains (VSG) emission in the surroundings of $\mathrm{HBe}$ stars. They suggested that the PAH/VSG emission from disks around B stars is absent. The observed emission emanates from the PDRs formed in the remnant of the parental cloud. They interpret this lack of emission as a sign that PAHs/VSGs have been photodissociated by hard UV photons in the disks. Since PAHs represent most of the UV optical depth, we conclude that this is also in good agreement with a scenario of photoevaporation in the disk around massive stars.

\section{Conclusions}

We briefly summarize the main conclusions of our study:

- We have carried out a search for circumstellar disks around $\mathrm{HBe}$ stars using the NRAO Very Large Array (VLA) and IRAM Plateau de Bure (PdB) interferometers. Interferometric observations are necessary to separate the disk from the surrounding envelope, although the disks themselves usually remain unresolved. Our goal is to investigate the properties of the circumstellar disks around intermediate mass stars to determine eventually their occurrence, lifetime, and evolution. Thus far, we have observed 6 objects with 4 successful detections.

- Our first result is that the disk mass is usually only a small percentage (less than 10\%) of the mass of the entire envelope. There are significant variations in the disk mass from source to source. Two disks of our sample, R Mon and $\mathrm{Z}$ CMa, have similar sizes to those found in TT and HAe stars. Z CMa is a FU Orionis object and cannot be directly compared with the others. The mass of the other large disk, $\mathrm{R}$ Mon, is a factor of 5 below that of an average HAe star. The disk around MWC 1080 is smaller ( $r_{\text {out }}<100$ AU) and less massive. Around MWC 297, we find an inner compact disk $\left(r_{\text {out }} \sim 29 \mathrm{AU}\right)$ and an outer ring at a distance of 200 AU. The $1.3 \mathrm{~mm} / 2.7 \mathrm{~mm}$ spectral index indicates that grain growth has proceeded in all disks in our sample.

- A comparison between our data and previous results in TT and HAe stars show that the masses of the circumstellar disks around $\mathrm{HBe}$ stars are on average 5-10 times lower than those around Herbig Ae stars. We propose that disk photoevaporation is responsible for this behavior. In HBe stars, the UV radiation disperses the gas in the outer disk on a timescale of a few $10^{5}$ yr. Once the outer part of the disk dissapears, the entire gaseous disk is photoevaporated on a short timescale $\left(\sim 10^{5} \mathrm{yr}\right)$, and only a dusty disk composed of large grains remains.

Acknowledgements. We thank the IRAM staff in Grenoble for their help and support during the observations and data reduction. This work was partly supported by INAF PRIN 2006 grant From disks to planetary systems. We thank the referee for his/her fruitful comments.

\section{References}

Acke, B., van den Ancker, M. E., Dullemond, C. P., van Boekel, R., \& Waters, L. B. F. M. 2004, A\&A, 422, 621

Acke, B., van den Ancker, M. E., \& Dullemond, C. P. 2005, A\&A, 436, 209 Acke, B., Verhoelst, T., van den Ancker, M. E., et al. 2008, A\&A, 485, 209 Alexander, R. 2008, New Astron. Rev., 52, 60

Alexander, R. D., \& Armitage, P. J. 2007, MNRAS, 375, 500

Alonso-Albi, T., Fuente, A., Bachiller, R., et al. 2007,

[arXiv: astro-ph/0702119]

Alonso-Albi, T., Fuente, A., Bachiller, R., et al. 2008, ApJ, 680, 1289

Berné, O., Joblin, C., Fuente, A., \& Ménard, F. 2009, A\&A, 495, 827

Cardelli, J. A., Clayton, G. C., \& Mathis, J. S. 1989, ApJ, 345, 245

Cesaroni, R., Neri, R., Olmi, L., et al. 2005, A\&A, 434, 1039

Close, L. M., Roddier, F., Hora, J. L., et al. 1997, ApJ, 489, 210

Dent, W. R. F., Matthews, H. E., \& Ward-Thompson, D. 1998, MNRAS, 301, 1049

di Francesco, J., Evans, II, N. J., Harvey, P. M., et al. 1997, ApJ, 482, 433

Dominik, C., Blum, J., Cuzzi, J. N., \& Wurm, G. 2007, in Protostars and Planets V, ed. B. Reipurth, D. Jewitt, \& K. Keil, 783

Draine, B. T. 2006, ApJ, 636, 1114

Dullemond, C. P., \& Dominik, C. 2004, A\&A, 417, 159

Eisner, J. A., Chiang, E. I., Lane, B. F., \& Akeson, R. L. 2007, ApJ, 657, 347

Eisner, J. A., Lane, B. F., Hillenbrand, L. A., Akeson, R. L., \& Sargent, A. I. 2004, ApJ, 613, 1049

Fuente, A., Rodríguez-Franco, A., Testi, L., et al. 2003, ApJ, 598, L39

Fuente, A., Alonso-Albi, T., Bachiller, R., et al. 2006, ApJ, 649, L119

Garaud, P. 2007, ApJ, 671, 2091

Grankin, K. N., Shevchenko, V. S., Chernyshev, A. V., et al. 1992, Informational Bulletin on Variable Stars, 3747, 1

Hartmann, L., Kenyon, S. J., Hewett, R., et al. 1989, ApJ, 338, 1001

Henning, T., \& Launhardt, R. 1998, A\&A, 338, 223

Henning, T., Launhardt, R., Steinacker, J., \& Thamm, E. 1994, A\&A, 291, 546

Hollenbach, D. J., Yorke, H. W., \& Johnstone, D. 2000, Protostars and Planets IV, 401

Johnson, H. L. 1966, ARA\&A, 4, 193

Koresko, C. D., Beckwith, S. V. W., Ghez, A. M., Matthews, K., \& Neugebauer, G. 1991, AJ, 102, 2073

Leinert, C., van Boekel, R., Waters, L. B. F. M., et al. 2004, A\&A, 423, 537

Mannings, V. 1994, MNRAS, 271, 587

Mannings, V., \& Sargent, A. I. 1997, ApJ, 490, 792

Mannings, V., \& Sargent, A. I. 2000, ApJ, 529, 391

Manoj, P., Ho, P. T. P., Ohashi, N., et al. 2007, ApJ, 667, L187

Meeus, G., Waters, L. B. F. M., Bouwman, J., et al. 2001, A\&A, 365, 476

Millan-Gabet, R., Schloerb, F. P., \& Traub, W. A. 2001, ApJ, 546, 358

Movsessian, T. A., Magakian, T. Y., \& Afanasiev, V. L. 2002, A\&A, 390, L5

Natta, A., Grinin, V. P., Mannings, V., \& Ungerechts, H. 1997, ApJ, 491, 885

Natta, A., Grinin, V., \& Mannings, V. 2000, Protostars and Planets IV, 559

Natta, A., Prusti, T., Neri, R., et al. 2001, A\&A, 371, 186

Ossenkopf, V., \& Henning, T. 1994, A\&A, 291, 943

Piétu, V., Dutrey, A., \& Kahane, C. 2003, A\&A, 398, 565

Piétu, V., Dutrey, A., Guilloteau, S., Chapillon, E., \& Pety, J. 2006, A\&A, 460, L43

Poetzel, R., Mundt, R., \& Ray, T. P. 1989, A\&A, 224, L13

Pontoppidan, K. M., Dullemond, C. P., Blake, G. A., et al. 2007, ApJ, 656, 980

Sandell, G., \& Weintraub, D. A. 2001, ApJS, 134, 115

Schaller, G., Schaerer, D., Meynet, G., \& Maeder, A. 1992, A\&AS, 96, 269

Schreyer, K., Semenov, D., Henning, T., \& Forbrich, J. 2006, ApJ, 637, L129

Siess, L., Dufour, E., \& Forestini, M. 2000, A\&A, 358, 593

Smith, J. D. T., Armus, L., Dale, D. A., et al. 2007, PASP, 119, 1133

Sylvester, R. J., Dunkin, S. K., \& Barlow, M. J. 2001, MNRAS, 327, 133

van den Ancker, M. E., Blondel, P. F. C., Tjin A Djie, H. R. E., et al. 2004, MNRAS, 349, 1516

Vink, J. S., Drew, J. E., Harries, T. J., \& Oudmaijer, R. D. 2002, MNRAS, 337, 356 


\section{Appendix A}

Table A.1. Table of fluxes for MWC 137.

\begin{tabular}{lllll}
\hline \hline Wavelength $(\mu m)$ & Flux $(\mathrm{Jy})$ & Flux error $(\mathrm{Jy})$ & Beam size $\left(^{\prime \prime}\right)$ & Reference / Flux type \\
\hline 0.440 & 0.019 & 0.001 & 0.8 & TYCHO-2 BAND_B \\
0.550 & 0.081 & 0.001 & 0.8 & TYCHO-2 BAND_V \\
0.700 & 0.017 & 0.003 & 0.9 & USNO-B BAND_R_JOHNSON_MORGAN \\
0.900 & 0.012 & 0.002 & 0.9 & USNO-B BAND_I_JOHNSON_MORGAN \\
1.235 & 0.499 & 0.010 & 2 & BAND_J_2MASS \\
1.620 & 0.428 & 0.001 & 1 & Morel (1978) BAND_H_JOHNSON_MORGAN \\
1.662 & 0.749 & 0.017 & 2 & BAND_H_2MASS \\
2.159 & 1.500 & 0.030 & 2 & BAND_Ks_2MASS \\
2.200 & 1.719 & 0.001 & 1 & Morel (1978) BAND_K_JOHNSON_MORGAN \\
3.400 & 3 & 0 & 1 & Morel (1978) BAND_L_JOHNSON_MORGAN \\
8.280 & 4.308 & 0.01 & 18.3 & BAND_A_MSX6C \\
12.000 & 10.9 & 0.1 & 30 & Hillenbrand (1992, IRAS) \\
12.130 & 4.371 & 0.010 & 18.3 & BAND_C_MSX6C \\
14.650 & 2.713 & 0.010 & 18.3 & BAND_D_MSX6C \\
21.340 & 4.834 & 0.010 & 18.3 & BAND_E_MSX6C \\
25.000 & 25.7 & 0.1 & 30 & Hillenbrand (1992, IRAS) \\
60.000 & 245.0 & 1.0 & 60 & Hillenbrand (1992, IRAS) \\
100 & 519.0 & 1.0 & 120 & Hillenbrand (1992, IRAS) \\
350 & 4 & 1 & 18.5 & Mannings (1994) \\
450 & 1.66 & 0.25 & 18.3 & Mannings (1994) \\
750 & 0.300 & 0.060 & 18.0 & Mannings (1994) \\
800 & 0.370 & 0.050 & 17.0 & Mannings (1994) \\
1100 & 0.095 & 0.014 & 18.8 & Mannings (1994) \\
1300 & 0.090 & 0.013 & 19.8 & Mannings (1994) \\
1400 & 0.0071 & $6 \times 10^{-4}$ & $3.05 \times 1.78$ & Our data \\
2700 & 0.0041 & $2.0 \times 10^{-4}$ & $6.2 \times 3.63$ & Our data \\
7000 & $2.3510^{-3}$ & $1.3 \times 10^{-4}$ & $2.21 \times 1.66$ & Our data \\
13000 & 0.0024 & $5 \times 10^{-4}$ & $5.18 \times 3.19$ & Our data \\
36000 & $5.7 \times 10^{-4}$ & $5 \times 10^{-5}$ & 0.3 & Skinner (1993) \\
60000 & $<6.0 \times 10^{-4}$ & - & 1.7 & Skinner (1993) \\
\hline & & & & \\
\hline
\end{tabular}

Table A.2. Extinction details for MWC 137.

\begin{tabular}{lllll}
\hline \hline $\begin{array}{l}\text { Wavelength } \\
(\mu \mathrm{m})\end{array}$ & $\begin{array}{l}\text { Extinction } \\
(\mathrm{mag})\end{array}$ & $\begin{array}{l}\text { Extinction from envelope model } \\
(\mathrm{mag})\end{array}$ & $\begin{array}{l}\text { Observed flux } \\
(\mathrm{mJy})\end{array}$ & $\begin{array}{l}\text { Dereddened flux } \\
(\mathrm{mJy})\end{array}$ \\
\hline 0.44 & 7.80 & 1.05 & 19.2 & 25340.3 \\
0.55 & 5.88 & 0.79 & 81.1 & 18294.1 \\
0.7 & 4.42 & 0.59 & 17.3 & 1013.9 \\
0.9 & 2.82 & 0.38 & 11.6 & 156.2 \\
1.235 & 1.69 & 0.23 & 498.5 & 2372.9 \\
1.62 & 1.09 & 0.15 & 428.1 & 1173.0 \\
1.662 & 1.05 & 0.14 & 748.7 & 1969.6 \\
2.159 & 0.69 & 0.09 & 1495.3 & 2821.1 \\
2.2 & 0.67 & 0.09 & 1719.3 & 3182.7 \\
\hline
\end{tabular}


Table A.3. Table of fluxes for LKH $\alpha 215$.

\begin{tabular}{lllll}
\hline \hline Wavelength $(\mu \mathrm{m})$ & Flux $(\mathrm{Jy})$ & Flux error $(\mathrm{Jy})$ & Beam size $\left(^{\prime \prime}\right)$ & Reference / Flux type \\
\hline 0.360 & 0.043 & 0.002 & 1 & Reed (2005) BAND_U_JOHNSON_MORGAN \\
0.440 & 0.110 & 0.001 & 1 & AC2000.2 BAND_B_JOHNSON_MORGAN \\
0.550 & 0.166 & 0.007 & 1 & Hipparcos BAND_V_JOHNSON_MORGAN \\
0.550 & 0.219 & 0.009 & 1 & Corporon (1999) BAND_V_JOHNSON_MORGAN \\
0.700 & 0.208 & 0.008 & 1 & Zacharias (2005) BAND_R_JOHNSON_MORGAN \\
1.235 & 0.580 & 0.012 & 2 & BAND_J_2MASS \\
1.620 & 0.328 & 0.001 & 1 & Morel (1978) BAND_H_JOHNSON_MORGAN \\
1.662 & 0.762 & 0.020 & 2 & BAND_H_2MASS \\
2.159 & 1.024 & 0.020 & 2 & BAND_Ks_2MASS \\
2.200 & 1.036 & 0.001 & 1 & Morel (1978) BAND_K_JOHNSON_MORGAN \\
3.400 & 1.280 & 0.001 & 1 & Morel (1978) BAND_L_JOHNSON_MORGAN \\
8.280 & 3.261 & 0.01 & 18.3 & BAND_A_MSX6C \\
12.000 & 6.3 & 0.1 & 30 & Hillenbrand (1992, IRAS) \\
12.130 & 4.2301 & 0.01 & 18.3 & BAND_C_MSX6C \\
14.650 & 1.84 & 0.01 & 18.3 & BAND_D_MSX6C \\
21.340 & 2.15 & 0.01 & 18.3 & BAND_E_MSX6C \\
25.000 & 8.2 & 0.1 & 30 & Hillenbrand (1992, IRAS) \\
60.000 & 86.9 & 0.1 & 60 & Hillenbrand (1992, IRAS) \\
100 & 315.0 & 1.0 & 120 & Hillenbrand (1992, IRAS) \\
450 & 1.23 & 0.22 & 18.3 & Mannings (1994) \\
800 & 0.142 & 0.020 & 17.0 & Mannings (1994) \\
1100 & 0.058 & 0.014 & 18.8 & Mannings (1994) \\
1400 & $<0.0010$ & - & $3.32 \times 1.84$ & Our data \\
2700 & $<3.0 \times 10^{-4}$ & - & $6.77 \times 3.68$ & Our data \\
\hline & & & & \\
\hline & & & & \\
\hline
\end{tabular}

Table A.4. Extinction details for LKH $\alpha 215$.

\begin{tabular}{lllll}
\hline \hline $\begin{array}{l}\text { Wavelength } \\
(\mu \mathrm{m})\end{array}$ & $\begin{array}{l}\text { Extinction } \\
(\mathrm{mag})\end{array}$ & $\begin{array}{l}\text { Extinction from envelope model } \\
(\mathrm{mag})\end{array}$ & $\begin{array}{l}\text { Observed flux } \\
(\mathrm{mJy})\end{array}$ & $\begin{array}{l}\text { Dereddened flux } \\
(\mathrm{mJy})\end{array}$ \\
\hline 0.36 & 5.37 & 0.78 & 42.7 & 6009.6 \\
0.44 & 4.54 & 0.66 & 109.5 & 7148.9 \\
0.55 & 3.42 & 0.50 & 166.3 & 3886.4 \\
0.55 & 3.42 & 0.50 & 219.2 & 5123.3 \\
0.70 & 2.57 & 0.37 & 208.2 & 2220.5 \\
1.235 & 0.99 & 0.14 & 579.8 & 1436.7 \\
1.62 & 0.64 & 0.09 & 327.8 & 589.0 \\
1.662 & 0.61 & 0.09 & 761.9 & 1337.3 \\
2.159 & 0.40 & 0.06 & 1024.1 & 1481.4 \\
2.20 & 0.39 & 0.06 & 1036.0 & 1482.1 \\
\hline
\end{tabular}


Table A.5. Table of fluxes for R Mon.

\begin{tabular}{|c|c|c|c|c|}
\hline Wavelength $(\mu \mathrm{m})$ & Flux (Jy) & Flux error (Jy) & Beam size $\left({ }^{\prime \prime}\right)$ & Reference/Flux type \\
\hline 1.235 & 0.213 & 0.005 & 2 & BAND_J_2MASS \\
\hline 1.256 & 0.027 & 0.0010 & 6 & Close $(\overline{1997)}$ \\
\hline 1.633 & 0.25 & 0.01 & 6 & Close (1997) \\
\hline 1.662 & 0.630 & 0.015 & 2 & BAND_H_2MASS \\
\hline 2.159 & 1.860 & 0.040 & 2 & BAND_Ks_2MASS \\
\hline 2.210 & 0.99 & 0.01 & 6 & Close (1997) \\
\hline 3.163 & 11.17 & 0.01 & 6 & Close (1997) \\
\hline 3.600 & 18 & 0 & 12 & Audard (2007) \\
\hline 3.930 & 19.69 & 0.01 & 6 & Close (1997) \\
\hline 4.290 & 21.05 & 0.01 & 18.3 & BAND_B1_MSX6C \\
\hline 4.350 & 29.16 & 0.01 & 18.3 & BAND_B2_MSX6C \\
\hline 4.674 & 23.85 & 0.01 & 6 & Close (1997) \\
\hline 5.800 & 30.41 & 0.4 & 12 & Audard (2007) \\
\hline 8.000 & 31 & 0 & 12 & Audard (2007) \\
\hline 8.280 & 32.09 & 0.01 & 18.3 & BAND_A_MSX6C \\
\hline 11.700 & 46.59 & 0.01 & 6 & Close $(\overline{1} 997)$ \\
\hline 12.000 & 58.3 & 0.1 & 30 & Hillenbrand (1992, IRAS) \\
\hline 12.130 & 47.18 & 0.01 & 18.3 & BAND_C_MSX6C \\
\hline 14.650 & 58.53 & 0.01 & 18.3 & BAND_D_MSX6C \\
\hline 20.800 & 103.1 & 0.1 & 6 & Close (1997) \\
\hline 21.340 & 102.2 & 0.01 & 18.3 & BAND_E_MSX6C \\
\hline 25.000 & 139.0 & 1.0 & 30 & Hillenbrand (1992, IRAS) \\
\hline 52.560 & 99 & 4 & 30 & Spitzer .tbl file \\
\hline 69.660 & 81 & 3 & 30 & Spitzer tbl file \\
\hline 93.600 & 62.3 & 2.0 & 30 & Spitzer .tbl file \\
\hline 350 & 2 & 0.300 & 18.5 & Mannings (1994) \\
\hline 450 & 1.56 & 0.25 & 18.3 & Mannings (1994) \\
\hline 800 & 0.220 & 0.040 & 17.0 & Mannings (1994) \\
\hline 1100 & 0.077 & 0.018 & 18.8 & Mannings (1994) \\
\hline 1200 & 0.049 & $10^{-4}$ & 11 & Our data - $30 \mathrm{~m}$ bolometer map \\
\hline 1300 & 0.079 & 0.019 & 19.8 & Mannings (1994) \\
\hline 1400 & 0.0118 & 0.0020 & $0.72 \times 0.33$ & Our data \\
\hline 2700 & 0.0041 & $5.0 \times 10^{-4}$ & $1.28 \times 0.83$ & Our data \\
\hline 7000 & $1.26 \times 10^{-3}$ & $1.3 \times 10^{-4}$ & $2.21 \times 1.66$ & Our data \\
\hline 13000 & $7.8 \times 10^{-4}$ & $1.0 \times 10^{-4}$ & $5.18 \times 3.19$ & Our data \\
\hline 20000 & $5.9 \times 10^{-4}$ & $3.0 \times 10^{-4}$ & 1.4 & Skinner (1993) \\
\hline 36000 & $4.0 \times 10^{-4}$ & $1.0 \times 10^{-4}$ & 0.3 & Skinner (1993) \\
\hline 60000 & $2.9 \times 10^{-4}$ & $1.0 \times 10^{-4}$ & 4.3 & Skinner (1993) \\
\hline
\end{tabular}

Table A.6. Extinction details for R Mon.

\begin{tabular}{lllll}
\hline \hline $\begin{array}{l}\text { Wavelength } \\
(\mu \mathrm{m})\end{array}$ & $\begin{array}{l}\text { Extinction } \\
(\mathrm{mag})\end{array}$ & $\begin{array}{l}\text { Extinction from envelope model } \\
(\mathrm{mag})\end{array}$ & $\begin{array}{l}\text { Observed flux } \\
(\mathrm{mJy})\end{array}$ & $\begin{array}{l}\text { Dereddened flux } \\
(\mathrm{mJy})\end{array}$ \\
\hline 1.235 & 0.66 & 1.36 & 212.9 & 390.8 \\
1.256 & 0.64 & 1.33 & 27.0 & 48.8 \\
1.633 & 0.42 & 0.87 & 250.0 & 368.3 \\
1.662 & 0.41 & 0.85 & 629.7 & 917.7 \\
2.159 & 0.27 & 0.55 & 1863.5 & 2386.2 \\
2.210 & 0.26 & 0.53 & 990.0 & 1256.2 \\
3.163 & 0.15 & 0.30 & 11170.0 & 12767.7 \\
\hline
\end{tabular}


Table A.7. Table of fluxes for Z CMa.

\begin{tabular}{|c|c|c|c|c|}
\hline Wavelength $(\mu \mathrm{m})$ & Flux (Jy) & Flux error $(\mathrm{Jy})$ & Beam size (") & Reference / Flux type \\
\hline 0.440 & 0.205 & 0.001 & 0.8 & TYCHO-2 BAND_B \\
\hline 0.550 & 0.474 & 0.001 & 0.8 & TYCHO-2 BAND_V \\
\hline 0.700 & 0.7 & 0.3 & 0.9 & USNO-B BAND_R_JOHNSON_MORGAN \\
\hline 0.900 & 0.9 & 0.4 & 0.9 & USNO-B BAND_I_JOHNSON_MORGAN \\
\hline 1.235 & 4 & 0 & 2 & BAND_J_2MASS \\
\hline 1.620 & 6 & 0 & 1 & Morel (1978) BAND_H_JOHNSON_MORGAN \\
\hline 1.662 & 8 & 0 & 2 & BAND_H_2MASS \\
\hline 2.159 & 21 & 3 & 2 & BAND_Ks_2MASS \\
\hline 2.200 & 25 & 0 & 1 & Morel (1978) BAND_K_JOHNSON_MORGAN \\
\hline 3.400 & 52 & 0 & 1 & Morel (1978) BAND_L_JOHNSON_MORGAN \\
\hline 3.800 & 54.0 & 4.0 & $<0.1$ & Koresko (1991) \\
\hline 4.290 & 71.78 & 0.01 & 18.3 & BAND_B1_MSX6C \\
\hline 4.350 & 72.12 & 0.01 & 18.3 & BAND_B2_MSX6C \\
\hline 4.800 & 69.0 & 6.0 & $<0.1$ & Koresko (1991) \\
\hline 8.280 & 107.7 & 0.1 & 18.3 & BAND_A_MSX6C \\
\hline 10.000 & 109.0 & 12.0 & $<0.1$ & Koresko (1991) \\
\hline 12.000 & 126.6 & 0.1 & 30 & Oudmaijer (1992, IRAS) \\
\hline 12.130 & 140.2 & 0.1 & 18.3 & BAND_C_MSX6C \\
\hline 14.650 & 159.1 & 0.1 & 18.3 & BAND_D_MSX6C \\
\hline 20.000 & 183.0 & 5.0 & 10 & ISO \\
\hline 21.340 & 202.8 & 0.1 & 18.3 & BAND_E_MSX6C \\
\hline 25.000 & 221.3 & 0.1 & 30 & Oudmaijer (1992, IRAS) \\
\hline 40.000 & 265.0 & 5.0 & 20 & ISO \\
\hline 50.000 & 340.0 & 10.0 & 30 & Elia (2004, ISO, chart estimate) \\
\hline 60.000 & 322.0 & 0.1 & 60 & Oudmaijer (1992, IRAS) \\
\hline 60.000 & 365.0 & 10.0 & 40 & Elia (2004, ISO, chart estimate) \\
\hline 70.000 & 390.0 & 10.0 & 40 & Elia (2004, ISO, chart estimate) \\
\hline 80.000 & 410.0 & 10.0 & 60 & Elia (2004, ISO, chart estimate) \\
\hline 90.000 & 420.0 & 10.0 & 80 & Elia (2004, ISO, chart estimate) \\
\hline 100 & 354.0 & 0.1 & 120 & Oudmaijer (1992, IRAS) \\
\hline 100 & 410.0 & 10.0 & 80 & Elia (2004, ISO, chart estimate) \\
\hline 120 & 390.0 & 10.0 & 80 & Elia (2004, ISO, chart estimate) \\
\hline 140 & 380.0 & 10.0 & 80 & Elia (2004, ISO, chart estimate) \\
\hline 160 & 310.0 & 10.0 & 80 & Elia (2004, ISO, chart estimate) \\
\hline 180 & 270.0 & 10.0 & 100 & Elia (2004, ISO, chart estimate) \\
\hline 350 & 29 & 1 & 19.0 & Dent (1998) \\
\hline 450 & 13.84 & 0.2 & 17.5 & Dent (1998) \\
\hline 450 & 11.41 & 0.1 & 8.0 & Sandell (2001) \\
\hline 800 & 1.964 & 0.013 & 16.0 & Dent (1998) \\
\hline 850 & 1.5 & 0.1 & 15.0 & Sandell (2001) \\
\hline 1100 & 0.710 & 0.030 & 18.7 & Dent (1998) \\
\hline 1300 & 0.83 & 0.01 & 21.0 & Sandell (2001) \\
\hline 1400 & 0.0260 & 0.0010 & $2.19 \times 0.85$ & Our data \\
\hline 2700 & 0.0085 & $7.0 \times 10^{-4}$ & $3.05 \times 1.41$ & Our data \\
\hline 6917 & 0.0021 & $1.0 \times 10^{-4}$ & $1.44 \times 1.43$ & Our data \\
\hline 13350 & 0.0021 & $1.0 \times 10^{-4}$ & $3.53 \times 2.71$ & Our data \\
\hline 36000 & 0.0022 & $3.0 \times 10^{-4}$ & $9.18 \times 6.56$ & Our data \\
\hline
\end{tabular}


Table A.8. Extinction details for Z CMa.

\begin{tabular}{lllll}
\hline \hline $\begin{array}{l}\text { Wavelength } \\
(\mu \mathrm{m})\end{array}$ & $\begin{array}{l}\text { Extinction } \\
(\mathrm{mag})\end{array}$ & $\begin{array}{l}\text { Extinction from envelope model } \\
(\mathrm{mag})\end{array}$ & $\begin{array}{l}\text { Observed flux } \\
(\mathrm{mJy})\end{array}$ & $\begin{array}{l}\text { Dereddened flux } \\
(\mathrm{mJy})\end{array}$ \\
\hline 0.44 & 5.09 & 0.0 & 204.6 & 22253.1 \\
0.55 & 3.84 & 0.0 & 474.0 & 16279.5 \\
0.7 & 2.88 & 0.0 & 689.6 & 9818.2 \\
0.9 & 1.84 & 0.0 & 890.4 & 4850.9 \\
1.235 & 1.11 & 0.0 & 3848.5 & 10654.0 \\
1.62 & 0.71 & 0.0 & 6075.0 & 11728.6 \\
1.662 & 0.69 & 0.0 & 8392.7 & 15778.0 \\
2.159 & 0.45 & 0.0 & 20774.5 & 31437.6 \\
2.2 & 0.44 & 0.0 & 24850.8 & 37144.3 \\
\hline
\end{tabular}

Table A.9. Table of fluxes for MWC 297.

\begin{tabular}{|c|c|c|c|c|}
\hline Wavelength $(\mu \mathrm{m})$ & Flux (Jy) & Flux error $(\mathrm{Jy})$ & Beam size $(")$ & Reference / Flux type \\
\hline 0.440 & 0.012 & 0.002 & 0.9 & USNO-B BAND_B_JOHNSON_MORGAN \\
\hline 0.550 & 0.046 & 0.002 & $<1$ & Eisner 2004 BAND_V_JOHNSON_MORGAN \\
\hline 0.700 & 0.300 & 0.060 & 0.9 & USNO-B BAND_R_JOHNSON_MORGAN \\
\hline 0.791 & 0.609 & 0.005 & 3 & BAND_I_DENIS \\
\hline 0.900 & 1.040 & 0.210 & 0.9 & USNO-B BAND_I_JOHNSON_MORGAN \\
\hline 1.228 & 8 & 0 & 3 & BAND_J_DENIS \\
\hline 1.235 & 6 & 0 & 2 & BAND_J_2MASS \\
\hline 1.620 & 6 & 0 & 1 & Morel (1978) BAND_H_JOHNSON_MORGAN \\
\hline 1.662 & 18 & 2 & 2 & BAND_H_2MASS \\
\hline 2.159 & 41 & 4 & 2 & BAND_Ks_2MASS \\
\hline 2.200 & 37 & 0 & 1 & Morel (1978) BAND_K_JOHNSON_MORGAN \\
\hline 3.400 & 78 & 0 & 1 & Morel (1978) BAND_L_JOHNSON_MORGAN \\
\hline 4.290 & 97.89 & 0.01 & 18.3 & BAND_B1_MSX6C \\
\hline 4.350 & 90.04 & 0.01 & 18.3 & BAND_B2_MSX6C \\
\hline 8.280 & 141.2 & 0.01 & 18.3 & BAND_A_MSX6C \\
\hline 12.000 & 159.0 & 1.0 & 30 & Hillenbrand (1992, IRAS) \\
\hline 12.130 & 124.7 & 0.01 & 18.3 & BAND_C_MSX6C \\
\hline 14.650 & 104.8 & 0.01 & 18.3 & BAND_D_MSX6C \\
\hline 21.340 & 114.2 & 0.01 & 18.3 & BAND_E_MSX6C \\
\hline 25.000 & 224.0 & 1.0 & 30 & Hillenbrand (1992, IRAS) \\
\hline 60.000 & 914.0 & 1.0 & 60 & Hillenbrand (1992, IRAS) \\
\hline 100 & 1800.0 & 10.0 & 120 & Hillenbrand (1992, IRAS) \\
\hline 350 & 4 & 0 & 18.5 & Mannings (1994) \\
\hline 450 & 2.46 & 0.13 & 18.3 & Mannings (1994) \\
\hline 600 & 0.970 & 0.110 & 17.5 & Mannings (1994) \\
\hline 750 & 0.840 & 0.060 & 18.0 & Mannings (1994) \\
\hline 800 & 0.699 & 0.013 & 17.0 & Mannings (1994) \\
\hline 850 & 0.681 & 0.022 & 18.0 & Mannings (1994) \\
\hline 1100 & 0.452 & 0.014 & 18.8 & Mannings (1994) \\
\hline 1300 & 0.432 & 0.025 & 19.8 & Mannings (1994) \\
\hline 1300 & 0.3 & 0.0 & $3.14 \times 3.02$ & Manoj (2007) \\
\hline 1300 & 0.175 & 0.005 & $1.1 \times 0.43$ & Our data \\
\hline 2600 & 0.149 & 0.0050 & $1.42 \times 0.88$ & Our data \\
\hline 6917 & 0.029 & 0.0010 & $1.95 \times 1.64$ & Our data \\
\hline 13350 & 0.0260 & $2.0 \times 10^{-4}$ & $3.92 \times 3.06$ & Our data \\
\hline 36000 & 0.01258 & $2.0 \times 10^{-4}$ & 7.2 & Skinner (1993) \\
\hline 60000 & 0.00917 & $2.0 \times 10^{-4}$ & 14.4 & Skinner (1993) \\
\hline
\end{tabular}


Table A.10. Extinction details for MWC 297.

\begin{tabular}{lllll}
\hline \hline $\begin{array}{l}\text { Wavelength } \\
(\mu \mathrm{m})\end{array}$ & $\begin{array}{l}\text { Extinction } \\
(\mathrm{mag})\end{array}$ & $\begin{array}{l}\text { Extinction from envelope model } \\
(\mathrm{mag})\end{array}$ & $\begin{array}{l}\text { Observed flux } \\
(\mathrm{mJy})\end{array}$ & $\begin{array}{l}\text { Dereddened flux } \\
(\mathrm{mJy})\end{array}$ \\
\hline 0.44 & 9.24 & - & 12.2 & 60645.3 \\
0.55 & 6.97 & - & 45.8 & 28036.0 \\
0.70 & 5.23 & - & 301.0 & 37286.4 \\
0.791 & 4.26 & - & 608.9 & 30758.9 \\
0.90 & 3.34 & - & 1041.4 & 22567.3 \\
1.228 & 2.02 & - & 7726.1 & 49860.6 \\
1.235 & 2.01 & - & 5645.3 & 35818.6 \\
1.62 & 1.30 & - & 6420.2 & 21181.2 \\
1.662 & 1.24 & - & 18009.4 & 56620.4 \\
2.159 & 0.82 & - & 40507.0 & 85900.8 \\
2.2 & 0.79 & - & 36926.7 & 76571.6 \\
\hline
\end{tabular}

Table A.11. Table of fluxes for MWC 1080.

\begin{tabular}{|c|c|c|c|c|}
\hline Wavelength $(\mu \mathrm{m})$ & Flux (Jy) & Flux error $(\mathrm{Jy})$ & Beam size $\left({ }^{\prime \prime}\right)$ & Reference / Flux type \\
\hline 0.440 & 0.022 & 0.001 & 0.8 & TYCHO-2 BAND_B \\
\hline 0.550 & 0.080 & 0.003 & $<1$ & Eisner 2004 BAND_V_JOHNSON_MORGAN \\
\hline 0.550 & 0.069 & 0.001 & 0.8 & TYCHO-2 BAND_V \\
\hline 0.700 & 0.120 & 0.024 & 0.9 & USNO-B BAND_R_JOHNSON_MORGAN \\
\hline 0.900 & 0.210 & 0.040 & 0.9 & USNO-B BAND_I_JOHNSON_MORGAN \\
\hline 1.235 & 1.650 & 0.030 & 2 & BAND_J_2MASS \\
\hline 1.620 & 1.921 & 0.001 & 1 & Morel (1978) BAND_H_JOHNSON_MORGAN \\
\hline 1.662 & 4 & 0 & 2 & BAND_H_2MASS \\
\hline 2.159 & 8 & 0 & 2 & BAND_Ks_2MASS \\
\hline 2.200 & 9 & 0 & 1 & Morel (1978) BAND_K_JOHNSON_MORGAN \\
\hline 3.400 & 17 & 0 & 1 & Morel (1978) BAND_L_JOHNSON_MORGAN \\
\hline 4.350 & 16.66 & 0.01 & 18.3 & BAND_B2_MSX6C \\
\hline 8.280 & 16.99 & 0.01 & 18.3 & BAND_A_MSX6C \\
\hline 12.130 & 17.91 & 0.01 & 18.3 & BAND_C_MSX6C \\
\hline 14.650 & 13.88 & 0.01 & 18.3 & BAND_D_MSX6C \\
\hline 21.340 & 16.12 & 0.01 & 18.3 & BAND_E_MSX6C \\
\hline 60.000 & 150.0 & 1.0 & 60 & Hillenbrand (1992, IRAS) \\
\hline 100 & 246.2 & 0.1 & 120 & Hillenbrand (1992, IRAS) \\
\hline 350 & $<24.55$ & - & 18.5 & Mannings (1994) \\
\hline 450 & 5 & 0 & 18.3 & Mannings (1994) \\
\hline 600 & 1.61 & 0.22 & 17.5 & Mannings (1994) \\
\hline 750 & 0.774 & 0.06 & 18.0 & Mannings (1994) \\
\hline 800 & 0.638 & 0.014 & 17.0 & Mannings (1994) \\
\hline 850 & 0.660 & 0.080 & 18.0 & Mannings (1994) \\
\hline 1100 & 0.250 & 0.030 & 18.8 & Mannings (1994) \\
\hline 1300 & 0.237 & 0.03 & 19.8 & Mannings (1994) \\
\hline 1400 & 0.0031 & $2.0 \times 10^{-4}$ & $1.28 \times 0.79$ & Our data \\
\hline 2700 & $<0.0017$ & - & $4.89 \times 2.75$ & Our data \\
\hline 36000 & $1.4 \times 10^{-4}$ & $2.0 \times 10^{-5}$ & 1.0 & Skinner (1993) \\
\hline
\end{tabular}


Table A.12. Extinction details for MWC 1080.

\begin{tabular}{lllll}
\hline \hline $\begin{array}{l}\text { Wavelength } \\
(\mu \mathrm{m})\end{array}$ & $\begin{array}{l}\text { Extinction } \\
(\mathrm{mag})\end{array}$ & $\begin{array}{l}\text { Extinction from envelope model } \\
(\mathrm{mag})\end{array}$ & $\begin{array}{l}\text { Observed flux } \\
(\mathrm{mJy})\end{array}$ & $\begin{array}{l}\text { Dereddened flux } \\
(\mathrm{mJy})\end{array}$ \\
\hline 0.44 & 6.16 & 4.24 & 21.9 & 6363.0 \\
0.55 & 4.64 & 3.19 & 79.6 & 5738.3 \\
0.55 & 4.64 & 3.19 & 68.7 & 4952.7 \\
0.70 & 3.49 & 2.40 & 119.8 & 2977.8 \\
0.90 & 2.23 & 1.53 & 209.7 & 1630.0 \\
1.235 & 1.34 & 0.92 & 1652.3 & 5662.8 \\
1.62 & 0.86 & 0.59 & 1921.1 & 4257.5 \\
1.662 & 0.83 & 0.57 & 4152.4 & 8911.5 \\
2.159 & 0.54 & 0.37 & 7825.8 & 12917.3 \\
2.20 & 0.53 & 0.36 & 8696.4 & 14141.4 \\
\hline
\end{tabular}

Table A.13. Compilation of disks masses ${ }^{1}$.

\begin{tabular}{|c|c|c|c|c|c|c|c|c|c|}
\hline " Name & $\begin{array}{l}\text { Spectral } \\
\text { type }\end{array}$ & $\begin{array}{l}\text { Luminosity } \\
\left(L_{\odot}\right)\end{array}$ & $\begin{array}{l}\text { Teff } \\
(\mathrm{K})\end{array}$ & $\begin{array}{l}\text { Distance } \\
(\mathrm{pc})\end{array}$ & $\begin{array}{l}\text { Star mass } \\
\left(M_{\odot}\right)\end{array}$ & $\begin{array}{l}\text { Age } \\
\text { (Myr) }\end{array}$ & $\begin{array}{l}\lambda \\
(\mathrm{mm})\end{array}$ & $\begin{array}{l}\begin{array}{l}\text { Flux } \\
(\mathrm{mJy})\end{array} \\
\text { (a) }\end{array}$ & $\begin{array}{l}\text { Disk mass } \\
\left(M_{\odot}\right)\end{array}$ \\
\hline AB Aur & A0 & 49 & 9500 & 144 & 2.57 & 3.87 & 1.4 & 85 & 0.0278 \\
\hline HD 100546 & B9 & 45 & 10500 & 103 & 2.53 & 5.59 & 1.3 & 465 & 0.0540 \\
\hline HD 142527 & F6 & 69 & 6300 & 200 & 3.54 & 1.11 & 1.3 & 1190 & 1.4860 \\
\hline HD 179218 / MWC 614 & B9 & 500 & 10000 & 240 & 5.02 & 0.473 & 1.3 & 71 & 0.0447 \\
\hline HD 100453 & A9 & 8 & 7400 & 114 & 1.66 & 14.1 & 1.2 & 270 & 0.0709 \\
\hline CD-36 10010B & F8 & 8 & 6200 & 84 & 1.63 & 9.49 & 1.3 & 142 & 0.0345 \\
\hline HD 139614 & A7 & 11 & 7800 & 157 & 1.83 & 10.7 & 1.3 & 242 & 0.1293 \\
\hline HD 169142 & B9 & 44 & 10500 & 145 & 2.5 & 5.69 & 1.3 & 169 & 0.0389 \\
\hline HD 142666 & A8 & 8 & 7500 & 116 & 1.65 & 14.9 & 1.2 & 79 & 0.0201 \\
\hline HD 144432 & A9 & 32 & 7300 & 200 & 2.26 & 3.87 & 1.3 & 37 & 0.0369 \\
\hline HD 150193 / MWC 863 & A1 & 30 & 9500 & 150 & 2.25 & 6.10 & 1.3 & 45 & 0.0143 \\
\hline HD 163296 & A1 & 36 & 9500 & 122 & 2.49 & 5.79 & 1.3 & 705 & 0.1485 \\
\hline HD 34282 & A0 & 36 & 9500 & 400 & 2.49 & 5.79 & 1.3 & 110 & 0.2259 \\
\hline HD 35187 & A2 & 34 & 9100 & 150 & 2.43 & 4.65 & 1.2 & 20 & 0.0055 \\
\hline UX Ori & A3 & 42 & 8600 & 430 & 2.43 & 4.22 & 1.2 & 19.8 & 0.0481 \\
\hline CQ Tau & F2 & 5 & 6800 & 100 & 1.49 & 769 & 1.3 & 143 & 0.0415 \\
\hline MWC 758 & A3 & 21 & 8500 & 200 & 2.17 & 6.26 & 1.3 & 72 & 0.0471 \\
\hline $\mathrm{LkH} \alpha 259$ & A9 & 107 & 7320 & 850 & 3.6 & 1.17 & 2.6 & $<6$ & $<0.722$ \\
\hline WW Vul & A3 & 43 & 8600 & 550 & 2.44 & 4.19 & 1.2 & 9.1 & 0.0362 \\
\hline BF Ori & A5 & 34 & 8300 & 450 & 2.34 & 4.37 & 1.3 & 6 & 0.0228 \\
\hline SV Cep & A0 & 14 & 8600 & 715 & 1.91 & 11.7 & 1.3 & 7 & 0.0459 \\
\hline V376 Cas & B5 & 190 & 13800 & 600 & 3.77 & 2.66 & 2.7 & $<5$ & $<0.089$ \\
\hline LkH $\alpha 198$ & B7 & 210 & 12000 & 600 & 3.51 & 1.49 & 2.7 & $<5$ & $<0.1141$ \\
\hline VY Mon & B8 & 1400 & 10700 & 800 & 6.9 & 0.243 & 1.3 & 120 & 0.6874 \\
\hline HD 97048 & A0 & 44 & 10000 & 150 & 2.53 & 4.61 & 1.3 & 452 & 0.1305 \\
\hline $\mathrm{LkH} \alpha 234$ & B5 & 800 & 14500 & 1250 & 5.01 & 0.524 & 1.3 & $<20$ & $<0.181$ \\
\hline T Ori & A3 & 83 & 8500 & 460 & 2.92 & 2.49 & 1.3 & $<6$ & $<0.021$ \\
\hline V380 Ori & B9 & 100 & 9500 & 460 & 3.05 & 2.00 & 1.3 & $<24$ & $<0.056$ \\
\hline $\mathrm{LkH} \alpha 218$ & B9 & 107 & 10700 & 1150 & 2.97 & 2.77 & 1.3 & $<27$ & $<0.3906$ \\
\hline Elias 3-1 & A6 & 21 & 8000 & 140 & 2.06 & 6.11 & 2.7 & 45 & 0.1360 \\
\hline $\mathrm{BD}+404124$ & B2 & 5900 & 22000 & 1000 & - & - & 2.7 & $<5$ & $<0.133$ \\
\hline V1686 Cyg & B5 & 1820 & 15500 & 1000 & 6.05 & 0.312 & 2.7 & $<5$ & $<0.246$ \\
\hline $\mathrm{BD}+61154$ & B8 & 225 & 11800 & 650 & 3.88 & 1.43 & 2.7 & 11.2 & 0.3509 \\
\hline RR Tau & A4 & 150 & 8800 & 800 & 3.57 & 1.34 & 1.3 & $<20$ & $<0.224$ \\
\hline HD 200775 & B2 & 1900 & 18500 & 600 & 6.9 & 0.337 & 1.3 & $<6$ & $<0.007$ \\
\hline MWC 480 & A3 & 25 & 8500 & 130 & 2.19 & 5.98 & 1.4 & 235 & 0.0797 \\
\hline HD 34700 & G0 & 20 & 6000 & 90 & 2.36 & 3.39 & 1.35 & 11.7 & 0.0037 \\
\hline MWC 297 & B1.5 & 10700 & 24000 & 250 & 9 & 1 & 1.3 & 168.6 & 0.0323 \\
\hline Z CMa & B8 & 9200 & 20000 & 930 & 12 & 0.05 & 1.4 & 24.1 & 0.2307 \\
\hline R Mon & B0 & 5600 & 25000 & 800 & 8 & 1 & 1.4 & 8.3 & 0.0093 \\
\hline MWC 1080 & B0 & 5600 & 25000 & 1000 & 10 & 1 & 1.4 & 2.1 & 0.0036 \\
\hline MWC 137 & B0 & 15000 & 26000 & 1300 & 14 & 1 & 1.4 & 7.1 & $<0.007$ \\
\hline VV Ser & A0 & 50 & 10000 & 260 & 2.68 & 3.87 & 1.3 & 1.44 & 0.0012 \\
\hline $\mathrm{LkH} \alpha 215$ & B7.5 & 5300 & 22000 & 800 & 7 & 0.4 & 1.4 & $<1.5$ & $<0.009$ \\
\hline
\end{tabular}

${ }^{1}$ The flux shown is the excess at $1.3 \mathrm{~mm}$ subtracting the free-free contribution in our sources. In MWC 297 we have used the flux of Manoj et al. (2007). References for the mm fluxes are: Acke et al. (2004), Alonso-Albi et al. (2008), di Francesco et al. (1997), Fuente et al. (2003), Fuente et al. (2006), Henning et al. (1994), Henning et al. (1998), Mannings et al. (1997), Mannings et al. (2000), Natta et al. (1997), Natta et al. (2001), Piétu et al. (2003), Piétu et al. (2006), Sylvester et al. (2001). 